TRANSACTIONS OF THE

AMERICAN MATHEMATICAL SOCIETY

Volume 355, Number 11, Pages 4397-4426

S 0002-9947(03)03291-4

Article electronically published on July 10, 2003

\title{
SHEAF COHOMOLOGY AND FREE RESOLUTIONS OVER EXTERIOR ALGEBRAS
}

\author{
DAVID EISENBUD, GUNNAR FLØYSTAD, AND FRANK-OLAF SCHREYER
}

\begin{abstract}
We derive an explicit version of the Bernstein-Gel'fand-Gel'fand (BGG) correspondence between bounded complexes of coherent sheaves on projective space and minimal doubly infinite free resolutions over its "Koszul dual" exterior algebra. Among the facts about the BGG correspondence that we derive is that taking homology of a complex of sheaves corresponds to taking the "linear part" of a resolution over the exterior algebra.

We explore the structure of free resolutions over an exterior algebra. For example, we show that such resolutions are eventually dominated by their "linear parts" in the sense that erasing all terms of degree $>1$ in the complex yields a new complex which is eventually exact.

As applications we give a construction of the Beilinson monad which expresses a sheaf on projective space in terms of its cohomology by using sheaves of differential forms. The explicitness of our version allows us to prove two conjectures about the morphisms in the monad, and we get an efficient method for machine computation of the cohomology of sheaves. We also construct all the monads for a sheaf that can be built from sums of line bundles, and show that they are often characterized by numerical data.
\end{abstract}

\section{INTRODUCTION}

Let $V$ be a finite-dimensional vector space over a field $K$, and let $W=V^{*}$ be the dual space. In this paper we will study complexes and resolutions over the exterior algebra $E=\bigwedge V$ and their relation to modules over $S=$ sym $W$ and sheaves on projective space $\mathbf{P}(W)$.

In this paper we study the Bernstein-Gel'fand-Gel'fand (BGG) correspondence [BGG, usually stated as an equivalence between the derived category of bounded complexes of coherent sheaves on $\mathbf{P}(W)$ and the stable category of finitely generated graded modules over $E$. Its essential content is a functor $\mathbf{R}$ from complexes of graded $S$-modules to complexes of graded $E$-modules, and its adjoint $\mathbf{L}$. For example, if $M=\bigoplus_{i} M_{i}$ is a graded $S$-module (regarded as a complex with just one term), then as a bigraded $E$-module $\mathbf{R}(M)=\operatorname{Hom}_{K}(E, M)$, with differential $\operatorname{Hom}_{K}\left(E, M_{i}\right) \rightarrow \operatorname{Hom}_{K}\left(E, M_{i+1}\right)$ defined from the multiplication map on $M$. Similarly, for a graded $E$-module $P$, we have $\mathbf{L}(P)=S \otimes_{K} P$. In fact (Proposition $2.1) \mathbf{R}$ is an equivalence from the category of graded $S$-modules to the category of linear complexes of free $E$-modules; here linear means essentially that the maps are represented by matrices of linear forms. A similar statement holds for $\mathbf{L}$.

Received by the editors December 1, 2001.

2000 Mathematics Subject Classification. Primary 14F05, 14Q20, 16E05.

The first and third authors are grateful to the NSF for partial support during the preparation of this paper. The third author wishes to thank MSRI for its hospitality. 
We show that finitely generated modules $M$ go to left-bounded complexes that are exact far to the right, and characterize the point at which exactness begins as the Castelnuovo-Mumford regularity of $M$. A strong form of this is Theorem 3.7, of which the following is a part:

Reciprocity Theorem. If $M$ is a graded $S$-module and $P$ is a graded E-module, then $\mathbf{R}(M)$ is an injective resolution of $P$ if and only if $\mathbf{L}(P)$ is a free resolution of $M$.

Let $\mathcal{F}$ be a coherent sheaf on projective space and take $M=\bigoplus_{d} \mathbf{H}^{0}(\mathcal{F}(d))$. The results above show that the complex $\mathbf{R}\left(M_{\geq r}\right)$ associated to the truncation of $M$ is acyclic for $r>>0$. If we take a minimal free resolution of the kernel of the first term in this complex, we obtain a doubly infinite exact free complex, independent of $r$, which we call the Tate resolution $\mathbf{T}(\mathcal{F})$ :

$$
\mathbf{T}(\mathcal{F}): \cdots \rightarrow T^{r-1} \rightarrow T^{r}=\operatorname{Hom}_{K}\left(E, M_{r}\right) \rightarrow \operatorname{Hom}_{K}\left(E, M_{r+1}\right) \rightarrow \cdots .
$$

It was first studied in Gel'fand Gel]. Our first main theorem (Theorem 4.1) is that the $e^{\text {th }}$ term of the Tate resolution is $T^{e}(\mathcal{F})=\bigoplus_{j} \operatorname{Hom}_{K}\left(E, \mathbf{H}^{j}(\mathcal{F}(e-j))\right)$; that is, it is made from the cohomology of the twists of $\mathcal{F}$. This leads to a new algorithm for computing sheaf cohomology. We have programmed this method in the computer algebra system Macaulay2 of Grayson and Stillman [http://www.math.uiuc.edu/ Macaulay2/]. In some cases it gives the fastest known computation of the cohomology.

We apply the Tate resolution to study a result of Beilinson Bei, which gives, for each sheaf $\mathcal{F}$ on projective space, a complex

$$
\cdots \rightarrow \bigoplus_{j=0}^{n} \mathbf{H}^{j}(\mathcal{F}(e-j)) \otimes_{K} \Omega_{\mathbf{P}^{n}}^{j-e}(j-e) \rightarrow \cdots
$$

called the Beilinson monad, whose homology is precisely $\mathcal{F}$ and whose terms depend only on the cohomology of a few twists of $\mathcal{F}$.

Our second main result is a constructive version of Beilinson's theorem [Bei], which clarifies its connection with the BGG-correspondence (Theorem 6.1). See Decker and Eisenbud [DE] for details and for an implementation of the BGG correspondence and the computation of the Beilinson monad. (That paper also contains an introduction to the uses of the Beilinson monad.)

Beilinson's original paper sketched a proof that leads easily to a weak form of the result, the "Beilinson spectral sequence", which determines the sheaf $\mathcal{F}$ only up to filtration. That version is explained in the book of Okonek, Schneider, and Spindler OSS. Kapranov Kap1 and Ancona and Ottaviani [AO] have given full proofs. However, their use of the derived category makes it difficult to compute the Beilinson monad effectively, and also makes it hard to obtain information about the maps in the monad.

Our construction of the Beilinson monad leads to new results about its structure. There are natural candidates for the linear components of the maps in the monad for a sheaf $\mathcal{F}$; and given such a monad, there are natural candidates for most of the maps in the monad of $\mathcal{F}(1)$. Our techniques allow us to prove that these natural candidates really do occur (Corollary 6.2 and Corollary 6.3).

A remarkable feature of the theory of resolutions over the exterior algebra, not visible for the corresponding theory over a polynomial ring, is that the linear terms of any resolution eventually predominate. To state this precisely, we introduce the 
linear part of a free complex $\mathbf{F}$ over $S$ or $E$. The linear part is the complex obtained from $\mathbf{F}$ by taking a minimal free complex $\mathbf{G}$ homotopic to $\mathbf{F}$, and then erasing all terms of absolute degree $>1$ from the matrices representing the differentials of $\mathbf{G}$. In fact, taking the linear part is functorial in a suitable sense: under the BGG correspondence it corresponds to the homology functor (Theorem 3.4). Just as the homology of a complex is simpler than the complex, one can often compute the linear part of a complex even when the complex itself is mysterious.

Of course free resolutions may have maps with no linear terms at all, that is, with linear part equal to zero. And they can have infinitely many maps with nonlinear terms unavoidably present (this is even the case for periodic resolutions). But the linear terms eventually predominate in the following sense:

Theorem 3.1. If $\mathbf{F}$ is the free resolution of a finitely generated module over the exterior algebra $E$, then the linear part of $\mathbf{F}$ is eventually exact.

This predominance can take arbitrarily long to assert itself: the resolution of the dual of the millionth syzygy of the residue field of $E$ has a million linear maps followed by a map with linear part 0 , and linear dominance happens only at the million and first term. In the case of a resolution of a monomial ideal, however, Herzog and Römer [HR] have shown that the linear part becomes exact after at most $\operatorname{dim}_{k} V$ steps. It would be interesting to know more results of this sort.

Beilinson [Bei] also proved the existence of a different monad for a sheaf $\mathcal{F}$, using the sheaves $\mathcal{O}_{\mathbf{P}}(i)$ for $0 \leq i \leq n=\operatorname{dim} \mathbf{P}(W)$ in place of the $\Omega^{i}(i)$. BernsteinGel'fand-Gel'fand also introduced a "linear" monad using sums of line bundles and only having maps given by matrices of linear forms. In the last section we show that such a monad "partitions" the cohomology of the sheaf into a "positive" part that appears as the homology of the corresponding complex of free $S$-modules and a "negative" part that appears as the cohomology of the dual complex. We explain how these and other free monads of a sheaf $\mathcal{F}$ arise from the Tate resolution $\mathbf{T}(\mathcal{F})$. We show that many such monads are characterized by simple numerical data.

Basic references for the BGG correspondence are Gel'fand Gel], and Gel'fandManin [GM]). Much of the elementary material of this paper could be done for an arbitrary pair of homogeneous Koszul algebras (in the sense of Priddy [Pri]) in place of the pair of algebras $S, E$. We use a tiny bit of this for the pair $(E, S)$. See Buchweitz [Buc1] for a sketch of the general case and a statement of general conditions under which the BGG correspondence holds. Buchweitz has also written a general treatment of the BGG correspondence over Gorenstein rings Buc2]. Versions of Beilinson's theorem have been established for some other varieties through work of Swan [Swa, Kapranov Kap1], Kap2, and Orlov Orl]. Yet other derived category equivalences have been pursued under the rubric of "tilting" (see Happel Hap ). Fløystad [Flo1] gives a general theory for Koszul pairs, and also studies how far the equivalences can be extended to unbounded complexes.

The material of our paper grew from two independent preprints of Eisenbud and Schreyer [ES1] and Fløystad [Flo2]. Since there was considerable overlap we wrote a more complete joint paper, which also includes new joint results. The original preprint by the second author has also been altered so that the notation and terminology are more aligned with the present paper.

The material in this paper has been applied to study the cohomology of hyperplane arrangements (Eisenbud, Popescu, and Yuzvinsky [EPY]) and to constructing counterexamples to the minimal free resolution conjecture for points in projective 
space (Eisenbud, Popescu, Schreyer, and Walter [EPSW]). The technique developed here for the Beilinson monad has been used by Eisenbud and Schreyer to construct complexes on various Grassmannians that can be used to compute and study Chow forms [ES2]. In a direction related to Green's proof of the linear syzygy conjecture [EW], Eisenbud and Weyman have found a general analogue for the Fitting lemma over $\mathbf{Z} / \mathbf{2}$-graded algebras, including the exterior algebra.

This paper owes much to the experiments we were able to make using the computer algebra system Macaulay2 of Grayson and Stillman, and we would like to thank them for their help and patience with this project. We are also grateful to Luchezar Avramov for getting us interested in resolutions over exterior algebras.

\section{Notation AND BACKGROUND}

Throughout this paper we write $K$ for a fixed field, and $V, W$ for dual vector spaces of finite dimension $v$ over $K$. We give the elements of $W$ degree 1 , so that the elements of $V$ have degree -1 . We write $E=\bigwedge V$ and $S=\operatorname{Sym}(W)$ for the exterior and symmetric algebras; these algebras are graded by their internal degrees, whereby $\operatorname{Sym}_{i}(W)$ has degree $i$ and $\bigwedge^{j} V$ has degree $-j$. We think of $E$ as $\operatorname{Ext}_{S}^{\bullet}(K, K)$ and $S$ as $\operatorname{Ext}_{E}^{\bullet}(K, K)$.

We will always write the index indicating the degree of a homogeneous component of a graded module as subscripts. Thus if $M=\bigoplus M_{i}$ is a graded module over $E$ or $S$, then $M_{i}$ denotes the component of degree $i$. We let $M(a)$ be the shifted module, so that $M(a)_{b}=M_{a+b}$. We write complexes cohomologically, with upper indices and differentials of degree +1 . Thus if

$$
\mathbf{F}: \cdots \rightarrow F^{i} \rightarrow F^{i+1} \rightarrow \cdots
$$

is a complex, then $F^{i}$ denotes the term of cohomological degree $i$. We write $\mathbf{F}[a]$ for the complex whose term of cohomological degree $j$ is $F^{a+j}$.

We will write $\omega_{S}=S \otimes_{K} \bigwedge^{v} W$ for the module associated to the canonical bundle of $\mathbf{P}(W)$; note that $\Lambda^{v} W$ is a vector space concentrated in degree $v$, so that $\omega_{S}$ is noncanonically isomorphic to $S(-v)$. Similarly, we set $\omega_{E}:=\operatorname{Hom}_{K}(E, K)=$ $E \otimes_{K} \bigwedge^{v} W$, which is noncanonically isomorphic to $E(-v)$. It is easy to check that for any graded vector space $D$ we have $\operatorname{Hom}_{K}(E, D) \cong \omega_{E} \otimes_{K} D$ as left $E$-modules. For any $E$-module $P$, we set $P^{*}:=\operatorname{Hom}_{K}(P, K)$.

We often use the fact that the exterior algebra is Gorenstein and finite dimensional over $K$, which follows from the fact that $\operatorname{Hom}_{K}(E, K) \cong E$ as above. As a consequence, the dual of any exact sequence is exact and the notions of free module, injective module, and projective module coincide.

We also use the notion of Castelnuovo-Mumford regularity. The most convenient definition for our purposes is that the Castelnuovo-Mumford regularity of a graded $S$-module $M=\bigoplus_{i} M_{i}$ is the smallest integer $r$ such that the truncation $M_{\geq r}=$ $\bigoplus_{i \geq r} M_{i}$ is generated by $M_{r}$ and has a linear free resolution - that is, all the maps in its free resolution are represented by matrices of linear forms. See for example Eisenbud-Goto EG] or Eisenbud Eis for a discussion. The regularity of a sheaf $\mathcal{F}$ on projective space (equal to the regularity of $\bigoplus_{d} \mathbf{H}^{0}(\mathcal{F}(d)$ ) if this module is finitely generated) can also be expressed as the minimal $r$ for which $\mathbf{H}^{i}(\mathcal{F}(r-i))=0$ for all $i>0$. 
A free complex over $E$ or a graded free complex over $S$ is called minimal if all its maps can be represented by matrices with entries in the appropriate maximal ideal. For example, any linear complex is minimal.

\section{The Bernstein-Gel'Fand-Gel'Fand Correspondence}

In this section we give a brief exposition of the main idea of Bernstein-Gel'fandGel'fand [BGG]: a construction of a pair of adjoint functors between the categories of complexes over $E$ and over $S$. However, we avoid a peculiar convention, used in the original, according to which the differentials of complexes over $E$ were not homomorphisms of $E$-modules.

Let $e_{i}$ and $x_{i}$ be dual bases of $V$ and $W$ respectively, so that $\sum_{i} x_{i} \otimes e_{i} \in$ $W \otimes_{K} V$ corresponds to the identity element under the isomorphism $W \otimes_{K} V=$ $\operatorname{Hom}_{K}(W, W)$. Let $A$ and $B$ be vector spaces. Giving a map $A \otimes_{K} W \rightarrow^{\alpha} B$ is the same as giving a map $A \rightarrow^{\alpha^{\prime}} B \otimes_{K} V$ (where the tensor products are taken over $K)$. For example, given $\alpha$ we set $\alpha^{\prime}(a)=\sum_{i} e_{i} \otimes \alpha\left(a \otimes x_{i}\right)$.

We begin with a special case that will play a central role. We regard a graded $S$-module $M=\bigoplus M_{d}$ as a complex with only one term, in cohomological degree 0 , and define $\mathbf{R}(M)$ to be the complex

$$
\begin{aligned}
\cdots \stackrel{\phi}{\longrightarrow} \operatorname{Hom}_{K}\left(E, M_{d}\right) \stackrel{\phi}{\longrightarrow} \operatorname{Hom}_{K}\left(E, M_{d+1}\right) \stackrel{\phi}{\longrightarrow} \cdots, \\
\phi: \alpha \mapsto\left[e \mapsto \sum_{i} x_{i} \alpha\left(e_{i} e\right)\right] .
\end{aligned}
$$

Here $\operatorname{Hom}_{K}\left(E, M_{d}\right)$ has cohomological index $d$, and a map $\alpha \in \operatorname{Hom}_{K}\left(E, M_{d}\right)$ has degree $t$ if it factors through the projection from $E$ onto $E_{d-t}$. Note that the complex $\mathbf{R}(M)$ is linear in a strong sense: the $d^{\text {th }}$ free module $\operatorname{Hom}_{K}\left(E, M_{d}\right) \cong$ $\omega_{E} \otimes M_{d}$ has socle in degree $d$; in particular, all the maps are represented by matrices of linear forms.

Proposition 2.1. The functor $\mathbf{R}$ is an equivalence between the category of graded left $S$-modules and the category of linear free complexes over $E$ (those for which the $d^{\text {th }}$ free module has socle in degree $d$ ).

Proof. A collection of maps $\mu_{d}: W \otimes_{K} M_{d} \rightarrow M_{d+1}$ defines a module structure on the graded vector space $\bigoplus M_{d}$ if and only if it satisfies a commutativity and associativity condition expressed by saying that, for each $d$, the composition of the multiplication maps

$$
W \otimes_{K}\left(W \otimes_{K} M_{d}\right) \rightarrow W \otimes_{K} M_{d+1} \rightarrow M_{d+2}
$$

factors through $\operatorname{Sym}_{2} W \otimes_{K} M_{d}$. Since $\bigwedge^{2} W$ is the kernel of $W \otimes_{K} W \rightarrow \operatorname{Sym}_{2} W$, this is the same as saying that the induced map $\bigwedge^{2} W \otimes_{K} M_{d} \rightarrow M_{d+2}$ is zero, or again that the map $\phi^{2}: \operatorname{Hom}_{K}\left(E_{v}, M_{d}\right) \rightarrow \bigwedge^{2} V \otimes_{K} \operatorname{Hom}_{K}\left(E_{v}, M_{d+2}\right)$ is zero. This last is equivalent to $\mathbf{R}(M)$ being a complex. As the whole construction is reversible, we are done.

As a first step in extending $\mathbf{R}$ to all complexes, we consider the case of a module regarded as a complex with a single term, but in arbitrary cohomological degree. Let $M$ be an $S$-module, regarded as a complex concentrated in cohomological degree 0 . Then $M[a]$ is a complex concentrated in cohomological degree $-a$, and we set

$$
\mathbf{R}(M[a])=\mathbf{R}(M)[a] .
$$


Now consider the general case of a complex of graded $S$-modules

$$
\mathbf{M}: \cdots \rightarrow M^{i} \rightarrow M^{i+1} \rightarrow \cdots .
$$

Applying $\mathbf{R}$ to each $M^{i}$, regarded as a complex concentrated in cohomological degree $i$, we get a double complex, and we define $\mathbf{R}(\mathbf{M})$ to be the total complex of this double complex. Thus $\mathbf{R}(\mathbf{M})$ is the total complex of

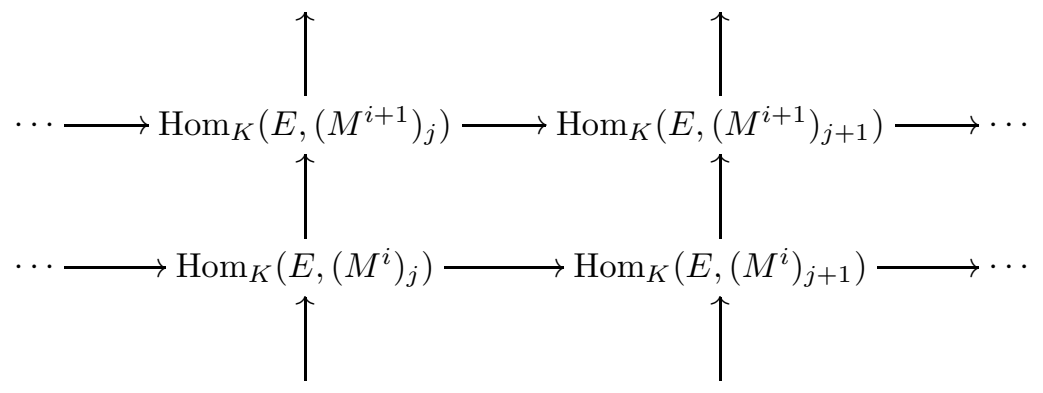

where the vertical maps are induced by the differential of $\mathbf{M}$ and the horizontal complexes are the complexes $\mathbf{R}\left(M^{i}\right)$ defined above. As $E$-modules we have

$$
(\mathbf{R M})^{k}=\sum_{i+j=k} \operatorname{Hom}_{K}\left(E,\left(M^{i}\right)_{j}\right),
$$

where $\left(M^{i}\right)_{j}$ is regarded as a vector space concentrated in degree $j$. Thus, as a bigraded $E$-module, $\mathbf{R}(\mathbf{M})=\operatorname{Hom}_{K}(E, \mathbf{M})$, and the formula for the graded components is

$$
\mathbf{R}(\mathbf{M})_{j}^{i}=\sum_{m} \operatorname{Hom}_{K}\left(E_{m-j},\left(M^{i-m}\right)_{m}\right) .
$$

The functor $\mathbf{R}$ has a left adjoint $\mathbf{L}$ defined in an analogous way by tensoring with $S$ : on a graded $E$-module $P=\sum P_{j}$ the functor $\mathbf{L}$ takes the value

$$
\mathbf{L}(P): \cdots \rightarrow S \otimes_{K} P_{j} \rightarrow S \otimes_{K} P_{j-1} \rightarrow \cdots,
$$

where the map takes $s \otimes p$ to $\sum_{i} x_{i} s \otimes e_{i} p$ and the term $S \otimes_{K} P_{j}$ has cohomological degree $-j$. If $\mathbf{P}$ is a complex of graded $E$-modules, then we can apply $\mathbf{L}$ to each term to get a double complex, and we define $\mathbf{L}(\mathbf{P})$ to be the total complex of this double complex, so that

$$
\mathbf{L}(\mathbf{P})^{k}=\sum_{i-j=k} S \otimes_{K}\left(P^{i}\right)_{j} \quad \text { and } \quad \mathbf{L}(\mathbf{P})_{j}^{i}=\sum_{m} S_{j-m} \otimes_{K}\left(P^{i+m}\right)_{m} .
$$

To see that $\mathbf{L}$ is the left adjoint of $\mathbf{R}$, we proceed as follows. First, if $M$ and $P$ are left modules over $S$ and $E$, respectively, then

$$
\operatorname{Hom}_{S}\left(S \otimes_{K} P, M\right)=\operatorname{Hom}_{K}(P, M)=\operatorname{Hom}_{E}\left(P, \operatorname{Hom}_{K}(E, M)\right) .
$$

If now $\mathbf{M}$ and $\mathbf{P}$ are complexes of graded modules over $S$ and $E$, we must prove that $\operatorname{Hom}_{S}(\mathbf{L}(\mathbf{P}), \mathbf{M}) \cong \operatorname{Hom}_{E}(\mathbf{P}, \mathbf{R}(\mathbf{M}))$, where on each side we take the maps of modules that preserve the internal and cohomological degrees and commute with the differentials. As a bigraded module, $\mathbf{L}(P)=S \otimes_{K} P$, and similarly for $\mathbf{R}$. Direct computation shows that these maps of complexes correspond to the maps of bigraded $K$-modules

$$
\phi=\left(\phi_{j}^{i}\right) \in \operatorname{Hom}_{\text {bigraded vector spaces }}(\mathbf{P}, \mathbf{M})
$$


such that $\phi_{j}^{i}: P_{j}^{i} \rightarrow M_{j}^{i-j}$ and

$$
\phi d-d \phi=\left(\sum_{s} x_{s} \otimes e_{s}\right) \phi
$$

where $\left(\sum_{s} x_{s} \otimes e_{s}\right) \phi$ takes an element $p \in P_{j}^{i}$ to $(-1)^{i} \sum_{s} x_{s} \phi\left(e_{s} p\right)$. We have proved:

Theorem 2.2 (Bernstein-Gel'fand-Gel'fand [BGG]). The functor $\mathbf{L}$, from the category of complexes of graded E-modules to the category of complexes of graded $S$-modules, is a left adjoint to the functor $\mathbf{R}$.

It is not hard to compute the homology of the complexes produced by $\mathbf{L}$ and $\mathbf{R}$ :

Proposition 2.3. If $M$ is a graded $S$-module and $P$ is a graded $E$-module, then

a) $\mathbf{H}^{i}(\mathbf{R}(M))_{j}=\operatorname{Tor}_{j-i}^{S}(K, M)_{j}$, and

b) $\mathbf{H}^{i}(\mathbf{L}(P))_{j}=\operatorname{Ext}_{E}^{j-i}(K, P)_{j}$.

Proof. The $j-i^{\text {th }}$ free module in the free resolution of $K$ over $E$ is $\left(\operatorname{Sym}_{j-i}(W)\right)^{*} \otimes_{K}$ $E$, which is generated by the vector space $\left(\operatorname{Sym}_{j-i}(W)\right)^{*}$ of degree $i-j$. We can use this to compute the right-hand side of the equality in $b)$ : the $j^{\text {th }}$ graded component of the module of homomorphisms of this into $P$ may be identified with $\operatorname{Sym}_{j-i}(W) \otimes_{K} P_{i}$. The differential is the same as that of $\mathbf{L}(P)$, and part $\left.b\right)$ follows. Part $a$ ) is similar (and even more familiar, from Koszul cohomology).

It follows that the exactness of $\mathbf{R}(M)$ or $\mathbf{L}(P)$ is a familiar condition. First the case of a module over the symmetric algebra:

Corollary 2.4. a) If $M$ is a finitely generated graded $S$-module, then the truncated complex

$$
\mathbf{R}(M)_{\geq d}: \operatorname{Hom}_{K}\left(E, M_{d}\right) \rightarrow \operatorname{Hom}_{K}\left(E, M_{d+1}\right) \rightarrow \cdots
$$

is acyclic (that is, has homology only at $\operatorname{Hom}_{K}\left(E, M_{d}\right)$ ) if and only if $M$ is $d$ regular.

Proof. By Proposition 2.3 applied to $M_{\geq d}$, the given sequence is acyclic if and only if $M_{\geq d}$ has linear free resolution.

Since any linear complex is of the form $\mathbf{L}(P)$ for a unique graded $E$-module $P$, it is perhaps most interesting to interpret part $b$ ) of Proposition 2.3 as a statement about linear complexes over $S$. The result below is implicitly used in Green's Gre] proof of the linear syzygy conjecture.

We call a right bounded linear complex

$$
\mathbf{G}: \cdots \rightarrow G^{-2} \rightarrow G^{-1} \rightarrow^{\phi} G^{0}
$$

irredundant if it is a subcomplex of the minimal free resolution of coker $(\phi)$ (or equivalently of any module whose presentation has linear part equal to $\phi$ ). (Eisenbud and Popescu in [EPo] called this property linear exactness, but to follow this usage would risk overusing the adjective "linear".)

Corollary 2.5. Let $\mathbf{G}$ be a minimal linear complex of free $S$-modules ending on the right with $G_{0}$ as above, and let $P^{*}$ be the E-module such that $\mathbf{L}\left(P^{*}\right)=\mathbf{G}$. The complex $\mathbf{G}$ is irredundant if and only the module $P$ is generated by $P_{0}$. The complex $\mathbf{G}$ is the linear part of a minimal free resolution if and only if the module $P$ is linearly presented. 
Proof. Let $\phi: G^{-1} \rightarrow G^{0}$ be the differential of $\mathbf{G}=\mathbf{L}\left(P^{*}\right)$, let

$$
\mathbf{F}: \cdots \rightarrow F^{-2} \rightarrow F^{-1} \stackrel{\phi}{\longrightarrow} G^{0}
$$

be the minimal free resolution of $\operatorname{coker}(\phi)$, and let $\kappa: \mathbf{G} \rightarrow \mathbf{F}$ be a comparison map lifting the identity on $G^{0}$. (This comparison map is unique, because $\mathbf{F}$ is minimal and $\mathbf{G}$ is linear.) By induction one sees that the comparison map is an injection if and only if $\mathbf{H}^{i} \mathbf{G}_{-i}=0$ for all $i<0$, and it is an isomorphism onto the linear part of $\mathbf{F}$ if and only if in addition $\mathbf{H}^{i} \mathbf{G}_{1-i}=0$ for all $i<0$. Proposition 2.3 shows that the first condition is satisfied if and only if $P^{*}$ injects into a direct sum of copies of $E$, while both conditions are true if and only if the minimal injective resolution begins with

$$
0 \rightarrow P^{*} \rightarrow \omega_{E}^{a} \rightarrow \omega_{E}(-1)^{b}
$$

for some numbers $a, b$. Dualizing, we get the desired linear presentation

$$
E(1)^{b} \rightarrow E^{a} \rightarrow P \rightarrow 0
$$

of $P$.

We now return to the BGG-correspondence. Both the functors $\mathbf{L}$ and $\mathbf{R}$ preserve mapping cones and homotopies of maps of complexes. For mapping cones this is immediate. For the second, note that two maps $f, g: \mathbf{F} \rightarrow \mathbf{G}$ of complexes are homotopic if and only if the induced map from $\mathbf{G}$ to the mapping cone of $f-g$ is split. This condition is preserved by any additive functor that preserves mapping cones.

Recall that a free resolution of a right bounded complex

$$
\mathbf{M}: \cdots \rightarrow M^{i-1} \rightarrow M^{i} \rightarrow M^{i+1} \rightarrow \cdots
$$

of graded $S$-modules is a graded free complex $\mathbf{F}$ with a morphism $\mathbf{F} \rightarrow \mathbf{M}$, homogeneous of degree 0, which induces an isomorphism on homology. We say that $\mathbf{F}$ is minimal if $K \otimes_{S} \mathbf{F}$ has trivial differential. Every right bounded complex $\mathbf{M}$ of finitely generated modules has a minimal free resolution, unique up to isomorphism. It is the minimal part of any free resolution.

The functors $\mathbf{L}$ and $\mathbf{R}$ give a general construction of resolutions.

Theorem 2.6. For any complex of graded $S$-modules $\mathbf{M}$, the complex $\mathbf{L R}(\mathbf{M})$ is a free resolution of $\mathbf{M}$ which surjects onto $\mathbf{M}$; and for any complex of graded E-modules $\mathbf{G}$, the complex $\mathbf{R} \mathbf{L}(\mathbf{G})$ is an injective resolution of $\mathbf{G}$ into which $\mathbf{G}$ injects.

In fact we shall see that every free complex whose homology is $M$ up to finite length comes as $\mathbf{L}$ of a complex that agrees with $\mathbf{R}(M)$ in high degrees.

An immediate consequence is:

Corollary 2.7. The functors $\mathbf{R}$ and $\mathbf{L}$ define an equivalence

$$
D^{b}(S \text {-mod }) \cong D^{b}(E-\bmod ) .
$$

Proof of Corollary 2.7. The derived category $D^{b}(S$-mod) of bounded complexes of finitely generated $S$-modules is equivalent to the derived category of complexes of finitely generated $S$-modules with bounded cohomology (that is, having just finitely many cohomology modules; see for example Hartshorne [Har, III, Lemma 12.3), and similarly for $E$. The functors $\mathbf{L}$ and $\mathbf{R}$ carry bounded complexes into complexes with bounded cohomology. This is clear for $\mathbf{L}$, and for $\mathbf{R}$ it follows from Corollary 
2.4. Thus $\mathbf{L}$ and $\mathbf{R}$ are well defined, and by Theorem $2.6 \mathbf{L R}$ and $\mathbf{R L}$ are both equivalent to the identity.

Proof of Theorem 2.6. The proofs of the two statements are similar, so we treat only the first. (A slight simplification is possible in the second case, since finitely generated modules over $E$ have finite composition series.)

Because $\mathbf{L}$ is the left adjoint functor of $\mathbf{R}$, there is a natural map $\mathbf{L R}(\mathbf{M}) \rightarrow \mathbf{M}$ adjoint to the identity map $\mathbf{R}(\mathbf{M}) \rightarrow \mathbf{R}(\mathbf{M})$. We claim that this is a surjective quasi-isomorphism.

To see that it is a surjection, consider a map $\phi: \mathbf{M} \rightarrow \mathbf{M}^{\prime}$ such that the composite $\mathbf{L R}(\mathbf{M}) \rightarrow \mathbf{M} \rightarrow \mathbf{M}^{\prime}$ is zero. It follows that the adjoint composition $\mathbf{R}(\mathbf{M}) \rightarrow \mathbf{R}(\mathbf{M}) \rightarrow \mathbf{R}\left(\mathbf{M}^{\prime}\right)$ is also zero, and since the first map is the identity, we get $\mathbf{R}(\phi)=0$. Since $\mathbf{R}$ is a faithful functor, $\phi=0$, proving surjectivity.

The functor $\mathbf{L}$ preserves direct limits because it is a left adjoint, while the functor $\mathbf{R}$ preserves direct limits because $E$ is a finite-dimensional vector space. Thus it suffices to prove our claim in the case where $\mathbf{M}$ is a bounded complex of finitely generated $S$-modules.

If $\mathbf{M}$ has the form

$$
\mathbf{M}: \cdots \rightarrow M^{d} \rightarrow 0 \rightarrow \cdots,
$$

then $\mathbf{M}$ admits $M^{d}[-d]$ (that is, the module $M^{d}$ considered as a complex concentrated in cohomological degree $d$ ) as a subcomplex, and the quotient is a complex of smaller length. Using the "five lemma", the naturality of the map $\mathbf{L R}(\mathbf{M}) \rightarrow \mathbf{M}$, and the exactness of the functor $\mathbf{L R}$, the claims will follow, by induction on the length of the complex, from the case where $\mathbf{M}$ has the form $M[-d]$ for some finitely generated graded $S$-module $M$ and integer $d$. This reduces immediately to the case $d=0$.

It thus suffices to see that $\mathbf{L R}(M) \rightarrow M$ is a quasi-isomorphism when $M$ is a finitely generated graded $S$-module. Now $\mathbf{R}(M)$ is the linear complex $\operatorname{Hom}_{K}\left(E, M_{0}\right)$ $\rightarrow \operatorname{Hom}_{K}\left(E, M_{1}\right) \rightarrow \cdots$, so $\mathbf{L R}(M)$ is the total complex of the following double complex:

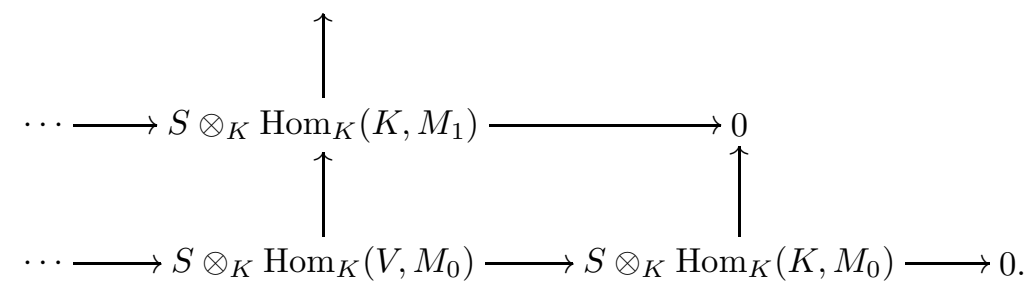

In this picture the terms below what is shown are all zero. The terms of cohomological degree 0 in the total complex are those along the diagonal going northwest from $S \otimes_{K} \operatorname{Hom}_{K}\left(K, M_{0}\right)$. The generators of $S \otimes_{K} \operatorname{Hom}_{K}\left(K, M_{0}\right)$ have internal degree 0, while those of $S \otimes_{K} \operatorname{Hom}_{K}\left(K, M_{1}\right)$ have internal degree 1, etc.

The $d^{\text {th }}$ row of this double complex is $S \otimes_{K} \operatorname{Hom}_{K}\left(E, M_{d}\right)$, which is equal to the complex obtained by tensoring the Koszul complex

$$
\cdots \rightarrow S \otimes_{K} \bigwedge^{2} W \rightarrow S \otimes_{K} W \rightarrow S \rightarrow 0
$$

with $M_{d}$. It is thus acyclic, its one cohomology module being $M_{d}$, in cohomological degree 0 . The spectral sequence starting with the horizontal cohomology of the double complex thus degenerates, and we see that the cohomology of the total 
complex $\mathbf{L R}(M)$ is a graded module with component of internal degree equal to $M_{d}$, concentrated in cohomological degree 0 . Thus $\mathbf{L R}(M)$ is acyclic, and the Hilbert function of $\mathbf{H}^{0}(\mathbf{L R}(M))$ is the same as that of $M$. As $\mathbf{L R}(M)$ has no terms in positive cohomological degree, and $M$ is in cohomological degree 0 , the surjection $\mathbf{L R}(M) \rightarrow M$ induces a surjection $\mathbf{H}^{0}(\mathbf{L R}(M)) \rightarrow M$, and we are done. (One can show that $\mathbf{L R}(M)$ is the tensor product, over $K$, of the Koszul complex and $M$, the action of $S$ being the diagonal action, but the isomorphism is complicated to write down.)

Though the statement of Theorem 2.6 has an attractive simplicity, it is not very useful in this form because the resolutions that are produced are highly nonminimal (for example, the free resolutions produced over $S$ are nearly always infinite). Theorem 3.7 shows that a modification of this construction gives at least an important part of the minimal free resolution.

\section{The Linear PART OF A COMPlex}

If $A$ is a matrix over $E$, then we define the linear part, written $\operatorname{lin}(A)$, to be the matrix obtained by erasing all the terms of entries of $A$ that are of degree $>1$. For example, if $a, b, c, d$ are linear forms of $E$, then the linear part of

$$
\left(\begin{array}{cc}
a & 0 \\
b c & d
\end{array}\right) \quad \text { is } \quad\left(\begin{array}{ll}
a & 0 \\
0 & d
\end{array}\right) \text {. }
$$

Taking the linear part is a functorial operation on maps (see Theorem 3.4 below), but taking the linear part of a matrix does not always commute with change of basis. For example, if $a, b, c$ are linear forms,

$$
d=\left(\begin{array}{cc}
a & 0 \\
0 & b
\end{array}\right), \quad \text { and } \quad e=\left(\begin{array}{ll}
1 & c \\
0 & 1
\end{array}\right),
$$

then $\operatorname{lin}(d e) \neq \operatorname{lin}(d) e$.

Suppose that $e: G \rightarrow H$ is a second map of free modules and that the composition $e d=0$. It need not be the case that $\operatorname{lin}(e) \operatorname{lin}(d)=0$; but if we assume in addition that $d(F)$ is in the maximal ideal times $G$ and $e(G)$ is in the maximal ideal times $H$, then $\operatorname{lin}(e) \operatorname{lin}(d)=0$ does follow. Thus, if $\mathbf{F}$ is a minimal free complex over $E$, we can define a new complex $\operatorname{lin}(\mathbf{F})$ by replacing each differential $d$ of $\mathbf{F}$ by its linear part, $\operatorname{lin}(d)$. Note that $\operatorname{lin}(\mathbf{F})$ is the direct sum of complexes $\mathbf{F}^{(i)}$ whose $e^{\text {th }}$ term is a direct sum of copies of $E(e+i)$ and whose maps are of degree 1 . In general, we define the linear part of any free complex $\mathbf{F}$ to be the linear part of a minimal complex homotopic to $\mathbf{F}$.

Theorem 3.1. Let $\mathbf{F}$ be a free or injective resolution of a finitely generated module over the exterior algebra $E$. The linear part of $\mathbf{F}$ is eventually exact.

Proof. We treat only the case where $\mathbf{F}$ is an injective resolution; by duality, the statement for a free resolution is equivalent. By Theorem 3.4 the linear part of $\mathbf{F}$ is the value of $\mathbf{R}$ on the $S$-module $\operatorname{Ext}_{E}^{\bullet}(K, P)$. Since any finitely generated $S$ module has finite regularity (see Eisenbud and Goto [EG]), it suffices by Corollary 2.4 to show that $\operatorname{Ext}_{E}^{\bullet}(K, P)$ is a finitely generated $S$-module. This was done by Aramova, Avramov, and Herzog [AAH]. For the reader's convenience we repeat the argument: we prove that $\operatorname{Ext}_{E}^{\bullet}(K, P)$ is a finitely generated $S$-module by induction 
on the length of $P$. If $P=K$, then $\operatorname{Ext}_{E}^{\bullet}(K, P)$ is free of rank 1 over $S$. If $P^{\prime}$ is a proper submodule of $P$, then from the exact sequence

$$
0 \rightarrow P^{\prime} \rightarrow P \rightarrow P / P^{\prime} \rightarrow 0
$$

we get an exact triangle of $S$-modules:

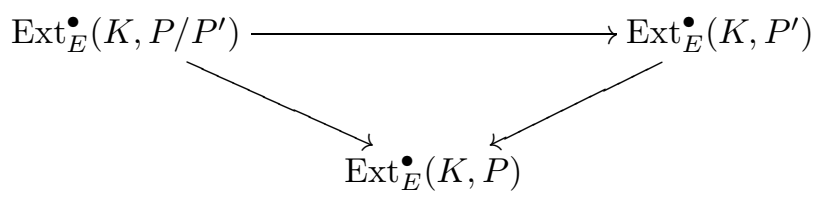

The two $S$-modules in the top row are finitely generated by induction, and thus $\operatorname{Ext}_{E}^{\bullet}(K, P)$ is finitely generated too.

If $P$ is an $E$-module, then we write $\operatorname{lin}(P)$ for the cokernel of $\operatorname{lin}(d)$, where $d$ is the map in a minimal free presentation of $P$. We can further define a family of modules connecting $P$ and $\operatorname{lin}(P)$ as follows: Let $d$ be a minimal free presentation of $P$, choose a representation of $d$ as a matrix, and let $e_{1}, \ldots, e_{v}$ be a basis of $V$. Let $d^{\prime}$ be the result of substituting $t e_{i}$ for $e_{i}$ in the entries of $d$, and then dividing each entry by $t$. The entries of $d$ have no constant terms because $d$ is minimal, and it follows that $d^{\prime}$ is a matrix over $K[t] \otimes_{K} E$. Let $P^{\prime}$ be the cokernel of $d^{\prime}$. It has fibers $P$ at $t \neq 0$ and $\operatorname{lin}(P)$ at 0 . The module $P^{\prime}$ may not be flat over $K[t]$, but the module $P^{\prime}\left[t^{-1}\right]$ is flat over $K\left[t, t^{-1}\right]$ : in fact, it is isomorphic to the module obtained from the trivial family $K\left[t, t^{-1}\right] \otimes_{K} P$ by pulling back along the automorphism $e_{i} \mapsto t e_{i} c$ of $E$.

Corollary 3.2. If $P$ is a finitely generated E-module, then any sufficiently high syzygy $Q$ of $P$ is a flat deformation of its linear part $\operatorname{lin}(Q)$.

Proof. If $Q$ is a sufficiently high syzygy, then by Theorem 3.1 the linear part of the minimal resolution of $Q$ is the resolution of $\operatorname{lin}(Q)$, so that (with the notation of the preceding paragraph) this free resolution of $Q$ lifts to a free resolution of $\operatorname{lin}\left(Q^{\prime}\right)$ over $K[t] \otimes_{K} E$. Thus $Q^{\prime}$ is flat, and the result follows.

Example 3.3. It is sometimes not so obvious what the linear part of the minimal version of a complex will be, and in particular it may be hard to read from the linear terms in a nonminimal version. For example, suppose that $W$ has dimension 2 and that $x, y \in W$ is a dual basis to $a, b \in V$. Consider the complex

$$
\mathbf{M}: 0 \rightarrow S /\left(x, y^{2}\right) \stackrel{x}{\longrightarrow} S /\left(x^{2}, y\right)(1) \rightarrow 0,
$$

where the notation means that the class of 1 goes to the class of $x$.

Applying $\mathbf{R}$ to $\mathbf{M}$, we get the double complex

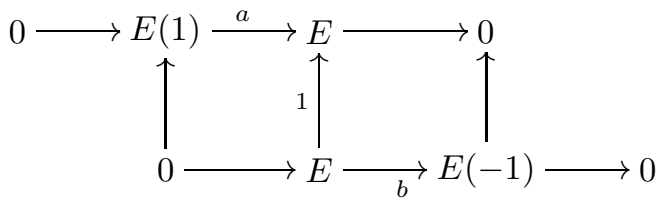

whose total complex is

$$
\mathbf{R}(\mathbf{M})=\mathbf{F}: \quad 0 \rightarrow E(1) \oplus E \stackrel{\left(\begin{array}{ll}
a & 1 \\
0 & b
\end{array}\right)}{\longrightarrow} E \oplus E(-1) \rightarrow 0 .
$$


Despite the presence of the linear terms in the differential of $\mathbf{F}$, the minimal complex $\mathbf{F}^{\prime}$ homotopic to $\mathbf{F}$ is

$$
\mathbf{F}^{\prime}: 0 \rightarrow E(1) \stackrel{a b}{\longrightarrow} E(-1) \rightarrow 0,
$$

so the differential of $\operatorname{lin}(\mathbf{F})$ is 0 .

Fortunately, we can construct the linear part of a complex directly and conceptually, without passing to a minimal complex or to matrices. First note that if $\mathbf{G}$ is a minimal free complex over $E$, then giving its linear part is equivalent, by Proposition 2.1, to giving the maps $\phi_{i}: \operatorname{Hom}_{E}\left(K, G^{i}\right) \rightarrow V \otimes_{K} \operatorname{Hom}_{E}\left(K, G^{i+1}\right)$ corresponding to the linear terms in the differential of $\mathbf{G}$. If $\mathbf{F}$ is a (possibly nonminimal) free complex homotopic to $\mathbf{G}$, then $\operatorname{Hom}_{E}\left(K, G^{i}\right)=\mathbf{H}^{i} \operatorname{Hom}_{E}(K, \mathbf{F})$. We will construct natural maps $\psi_{i}: \mathbf{H}^{i} \operatorname{Hom}_{E}(K, \mathbf{F}) \rightarrow V \otimes \mathbf{H}^{i+1} \operatorname{Hom}_{E}(K, \mathbf{F})$, and prove that $\psi_{i}=\phi_{i}$.

We identify $S$ with $\operatorname{Ext}_{E}(K, K)$ and use the well-known $\operatorname{Ext}_{E}(K, K)$-module structure on $\mathbf{H}^{\bullet} \operatorname{Hom}_{E}(K, \mathbf{F})$. To formulate this explicitly, we make use of the exact sequence

$$
\eta: 0 \rightarrow V \rightarrow E /(V)^{2} \rightarrow K \rightarrow 0 .
$$

The extension class

$$
\eta \in \operatorname{Ext}_{E}^{1}(K, V)=\operatorname{Ext}_{E}^{1}(K, K) \otimes_{K} V=\operatorname{Hom}_{K}\left(W, \operatorname{Ext}_{E}^{1}(K, K)\right)
$$

corresponds to the inclusion $W=\operatorname{Sym}_{1} W \subset \operatorname{Sym} W$. Since $\mathbf{F}$ is a free complex, the sequence $\operatorname{Hom}(\eta, \mathbf{F})$ is an exact sequence of complexes, and we obtain the homomorphism $\psi_{i}: \mathbf{H}^{i} \operatorname{Hom}_{E}(K, \mathbf{F}) \rightarrow V \otimes \mathbf{H}^{i+1} \operatorname{Hom}_{E}(K, \mathbf{F})$ from the connecting homomorphism

$$
\delta_{i}: W \otimes_{K} \mathbf{H}^{i} \operatorname{Hom}_{E}(K, \mathbf{F})=\mathbf{H}^{i} \operatorname{Hom}_{E}(V, \mathbf{F}) \rightarrow \mathbf{H}^{i+1} \operatorname{Hom}_{E}(K, \mathbf{F}) .
$$

Theorem 3.4. If $\mathbf{F}$ is a complex of free modules over $E$, then

$$
\operatorname{lin}(\mathbf{F})=\mathbf{R}\left(\mathbf{H}^{\bullet} \operatorname{Hom}_{E}(K, \mathbf{F})\right),
$$

where the $S$-module structure on $\mathbf{H}^{\bullet} \operatorname{Hom}_{E}(K, \mathbf{F})$ is given by the Yoneda action of $\operatorname{Ext}_{E}(K, K)$.

Proof. We use the notation $\phi_{i}, \psi_{i}, \delta_{i}$ introduced just before the theorem. From the definition of $\psi_{i}: \mathbf{H}^{i} \operatorname{Hom}_{E}(K, \mathbf{F}) \rightarrow V \otimes_{K} \mathbf{H}^{i+1} \operatorname{Hom}_{E}(K, \mathbf{F})$ we see that it depends only on the homotopy class of $\mathbf{F}$, so we may assume that $\mathbf{F}$ is minimal. By Proposition 2.1 we may assume that

$$
F^{i}=\operatorname{Hom}_{K}\left(E, M_{i}\right)=E \otimes_{K} \operatorname{Hom}_{K}\left(E_{v}, M_{i}\right) .
$$

Let $1 \otimes a \in K \otimes_{K} \operatorname{Hom}_{K}\left(E_{v}, M_{i}\right)$ be a generator which is mapped by $\phi_{i}$ to $\sum v_{j} \otimes$ $b_{j} \in V \otimes_{K} \operatorname{Hom}_{K}\left(E_{v}, M_{i+1}\right)$. Let $s=v_{0} \wedge v_{1} \wedge \cdots \wedge v_{n} \in E_{v}$ be a generator of the socle of $E$. To prove $\psi_{i}=\phi_{i}$ we have to show that an element of the form $w \otimes s \otimes a \in W \otimes_{K} E_{v} \otimes \operatorname{Hom}_{K}\left(E_{v}, M_{i}\right)=W \otimes_{K} \operatorname{Hom}_{E}\left(K, F^{i}\right)=\operatorname{Hom}_{E}\left(V, F^{i}\right)$ is mapped to $\left\{1 \mapsto \sum_{j} s w\left(v_{j}\right) \otimes b_{j}\right\} \in E_{v} \otimes_{K} \operatorname{Hom}_{K}\left(E_{v}, M_{i+1}\right)=\operatorname{Hom}_{E}\left(K, F^{i+1}\right)$ under the connecting homomorphism $\delta_{i}$.

The element $w \otimes s \otimes a$ corresponds to $\{v \mapsto s w(v) \otimes a\}$ in $\operatorname{Hom}_{E}\left(V, F^{i}\right)$. We must lift $\{v \mapsto s w(v) \otimes a\}$ to an element of $\operatorname{Hom}_{E}\left(E /(V)^{2}, F^{i}\right)$. The image of $1 \in E /(V)^{2}$ will be an element $c \in F^{i}=E \otimes_{K} \operatorname{Hom}_{K}\left(E_{v}, M_{i}\right)$ which satisfies $v \cdot c=s \otimes w(v) a$ for all $v \in V$, and any such element defines a lifting. We can take $c=s \neg w \otimes a$. The image of $w \otimes s \otimes a$ under the connecting homomorphism is the 
$\operatorname{map}\{1 \mapsto d(c)\} \in \operatorname{Hom}_{E}\left(K, F^{i+1}\right)$, where $d: F^{i} \rightarrow F^{i+1}$ is the differential of $\mathbf{F}$. With

$$
d(c)=s \neg w \wedge d(a)=s \neg w \wedge\left(\sum_{j} v_{j} \otimes b_{j}+\text { higher terms }\right)=\sum_{j} s w\left(v_{j}\right) \otimes b_{j}
$$

we arrive at the desired formula.

To understand the linear parts of complexes obtained from the functor $\mathbf{R}$, we will employ a general result: if the vertical differential of a suitable double complex splits, then the associated total complex is homotopic to one built from the homology of the vertical differential in a simple way.

Lemma 3.5. Let $\mathbf{F}$ be a double complex

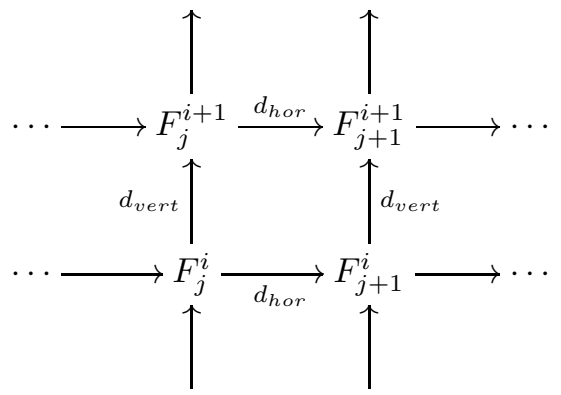

in some abelian category such that $F_{j}^{i}=0$ for $i \ll 0$. Suppose that the vertical differential of $\mathbf{F}$ splits, so that for each $i, j$ there is a decomposition $F_{j}^{i}=G_{j}^{i} \oplus$ $d_{\text {vert }} G_{j}^{i-1} \oplus H_{j}^{i}$ such that the kernel of $d_{\text {vert }}$ in $F_{j}^{i}$ is $H_{j}^{i} \oplus d_{\text {vert }} G_{j}^{i-1}$, and such that $d_{\text {vert }}$ maps $G_{j}^{i-1}$ isomorphically to $d_{\text {vert }}\left(G_{j}^{i}\right)$. If we write $\sigma: F_{j}^{i} \rightarrow H_{j}^{i}$ for the projection corresponding to this decomposition, and $\pi: F_{j}^{i} \rightarrow d_{\text {vert }} G_{j}^{i-1} \rightarrow G_{j}^{i-1}$ for the composition of the projection with the inverse of $d_{v e r t}$ restricted to $G_{j}^{i-1}$, then the total complex of $\mathbf{F}$ is homotopic to the complex

$$
\cdots \rightarrow \bigoplus_{i+j=k} H_{j}^{i} \stackrel{d}{\longrightarrow} \bigoplus_{i+j=k+1} H_{j}^{i} \rightarrow \cdots
$$

with differential

$$
d=\sum_{\ell \geq 0} \sigma\left(d_{h o r} \pi\right)^{\ell} d_{h o r} .
$$

Proof. We write $d_{\text {tot }}=d_{\text {vert }} \pm d_{\text {hor }}$ for the differential of the total complex. Note first that $\sigma\left(d_{h o r} \pi\right)^{\ell} d_{h o r}$ takes $H_{j}^{i}$ to $H_{j+1+\ell}^{i-\ell}$. Since $F_{j+1+\ell}^{i-\ell}=0$ for $\ell>>0$, the sum defining $d$ is finite.

Let $F$ denote $\mathbf{F}$ without the differential, that is, as a bigraded module. We will first show that $F$ is the direct sum of the three components

$$
G=\bigoplus_{i, j} G_{j}^{i}, \quad d_{t o t} G, \quad \text { and } H=\bigoplus_{i, j} H_{j}^{i},
$$

and that $d_{\text {tot }}$ is a monomorphism on $G$.

The same statements, with $d_{t o t}$ replaced by $d_{v e r t}$, are true by hypothesis. In particular, any element of $F$ is a sum of elements of the form $g^{\prime}+d_{\text {vert }} g+h$ with $g^{\prime} \in G_{j}^{i}, g \in G_{j}^{i-1}$ and $h \in H_{j}^{i}$ for some $i, j$. Modulo $G+d_{t o t} G+H$ this element 
can be written as $d_{h o r}(g) \in F_{j+1}^{i-1}$. As $F_{t}^{s}=0$ for $s<<0$, we may do induction on $i$, and assume that $d_{\text {hor }} g \in G+d_{\text {tot }} G+H$, so we see that $F=G+d_{t o t} G+H$.

Suppose

$$
g^{\prime} \in G=\bigoplus_{i+j=\ell} G_{j}^{i}, \quad g \in G=\bigoplus_{i+j=\ell-1} G_{j}^{i}, \quad h \in H=\bigoplus_{i+j=\ell} H_{j}^{i}
$$

and $g^{\prime}+d_{t o t} g+h=0$; we must show that $g=g^{\prime}=h=0$. Write $g=\sum_{k=a}^{b} g_{\ell-k}^{k-1}$ with $g_{t}^{s} \in G_{t}^{s}$. If $b-a=-1$, then $d_{t o t}=0$, and the desired result is a special case of the hypothesis. In any case, there is no component of $g$ in $G_{\ell-b-1}^{b}$, so the component of $d_{t o t} g$ in $G_{\ell-b}^{b}$ is equal to $d_{v e r t} g_{\ell-b}^{b-1}$. From the hypothesis we see that $d_{v e r t} g_{\ell-b}^{b-1}=0$, so $g_{\ell-b}^{b-1}=0$, and we are done by induction on $b-a$. This shows that $F=G \oplus d_{t o t} G \oplus H$ and that $d_{t o t}$ is an isomorphism from $G$ to $d_{t o t} G$.

The modules $G \oplus d_{t o t} G$ form a double complex contained in $\mathbf{F}$ that we will call $\mathbf{G}$. Since $d_{t o t}: G \rightarrow d_{t o t} G$ is an isomorphism, the total complex $t o t(\mathbf{G})$ is split exact. It follows that the total complex $\operatorname{tot}(\mathbf{F})$ is homotopic to $\operatorname{tot}(\mathbf{F}) / \operatorname{tot}(\mathbf{G})$, and the modules of this last complex are isomorphic to $\bigoplus_{i+j=k} H_{j}^{i}$. We will complete the proof by showing that the induced differential on $\operatorname{tot}(\mathbf{F}) / \operatorname{tot}(\mathbf{G})$ is the differential $d$ defined above.

Choose $h \in H_{j}^{i}$. The image of $h$ under the induced differential is the unique element $h^{\prime} \in H$ such that $d_{\text {tot }} h \equiv h^{\prime}(\bmod G+d G)$. Now

$$
d_{\text {tot }} h=d_{\text {hor }} h \equiv \sigma d_{\text {hor }} h+\left(d_{\text {vert }} \pi\right) d_{\text {hor }} h(\bmod G) .
$$

However,

$$
d_{\text {vert }} \pi \equiv d_{h o r} \pi \equiv \sigma\left(d_{\text {hor }} \pi\right)+d_{\text {vert }} \pi\left(d_{\text {hor }} \pi\right)\left(\bmod G+d_{\text {tot }} G\right) .
$$

Continuing this way, and using again the fact that $F_{j}^{i}=0$ for $i<<0$, we obtain

$$
d_{t o t} h \equiv \sum_{\ell} \sigma\left(d_{h o r} \pi\right)^{\ell} d_{h o r} h\left(\bmod G+d_{t o t} G\right),
$$

as required.

We apply Theorem 3.4 to complexes of the form $\mathbf{R}(\mathbf{M})$ :

Corollary 3.6. If $\mathbf{M}$ is a left-bounded complex of graded $S$-modules, then

$$
\operatorname{lin}(\mathbf{R}(\mathbf{M}))=\bigoplus_{i} \mathbf{R}\left(\mathbf{H}^{i}(\mathbf{M})\right)
$$

where $\mathbf{H}^{i}(\mathbf{M})$ is regarded as a complex of one term, concentrated in cohomological degree $i$. A similar statement holds for the linear part of $\mathbf{L}(\mathbf{P})$ when $\mathbf{P}$ is a leftbounded complex of graded E-modules.

Proof. As $\mathbf{M}$ is a left-bounded complex of finitely generated modules, the double complex whose total complex is $\mathbf{R}(\mathbf{M})$ satisfies the conditions of Lemma 3.5. The bigraded module underlying $\mathbf{R}\left(\mathbf{H}^{i}(\mathbf{M})\right)$ is precisely the module $H$ of Lemma 3.5, and the differential is the map $\sigma d_{h o r}$ restricted to $H$. This is a linear map. But the other terms in the sum $d=\sum_{\ell} \sigma\left(\pi d_{h o r}\right)^{\ell} d_{h o r}$ all involve two or more iterations of $d_{h o r}$, and are thus represented by matrices whose entries have degree at least 2 .

Example 3.3, continued. Note that the homology of $\mathbf{M}$ is $\mathbf{H}^{\bullet}(\mathbf{M})=K(-1) \oplus$ $K(1)[-1]$. We may write $\operatorname{lin}\left(\mathbf{F}^{\prime}\right)=\mathbf{R}(K(-1)) \oplus \mathbf{R}(K(1))[-1]$, as required by Corollary 3.6. 
Here is the promised information about the minimal resolution of a module:

Theorem 3.7. a) Reciprocity: If $M$ is a finitely generated graded $S$-module and $P$ is a finitely generated graded E-module, then $\mathbf{L}(P)$ is a free resolution of $M$ if and only if $\mathbf{R}(M)$ is an injective resolution of $P$.

b) More generally, for any minimal bounded complex of finitely generated graded $S$-modules $\mathbf{M}$, the linear part of the minimal free resolution of $\mathbf{M}$ is $\mathbf{L}(\mathbf{H} \bullet(\mathbf{R}(\mathbf{M}))$ ); and for any minimal bounded complex of finitely generated graded E-modules $\mathbf{P}$, the linear part of the minimal injective resolution of $\mathbf{P}$ is $\mathbf{R}\left(\mathbf{H}^{\bullet}(\mathbf{L}(\mathbf{P}))\right.$ ).

Proof. The two parts of $b$ ) being similar, we prove only the first statement. By Theorem 2.6 the complex $\mathbf{L R}(\mathbf{M})$ is a free resolution. The complex $\mathbf{R}(\mathbf{M})$ is leftbounded because $\mathbf{F}$ is bounded and contains only finitely generated modules. Thus we may apply Corollary 3.6, proving the first statement.

For the reciprocity statement $a$ ), suppose that $\mathbf{L}(P)$ is a minimal free resolution of $M$. By part $b$ ) the linear part of the minimal injective resolution of $P$ is $\mathbf{R}\left(\mathbf{H}^{\bullet}(\mathbf{L}(P))\right)$. Since $\mathbf{L}(P)$ is a resolution of $M$, this is $\mathbf{R}(M)$. All the terms of cohomological degree $d$ of this complex have degree $-d$, so there is no room for nonlinear differentials, and the linear part of the resolution is the resolution.

\section{Sheaf COHOMOlOgY AND EXTERIOR SYZYGIES}

In this section we establish a formula for the free modules that appear in resolutions over $E$. Because $E$ is Gorenstein, it is natural to work with doubly infinite resolutions:

A Tate resolution over $E$ is a doubly infinite free complex

$$
T: \cdots \rightarrow T^{d} \rightarrow T^{d+1} \rightarrow \cdots
$$

that is everywhere exact.

There is a Tate resolution naturally associated to a coherent sheaf $\mathcal{F}$ on $\mathbf{P}(W)$, defined as follows. Let $M$ be a finitely generated graded $S$-module representing $\mathcal{F}$, for example $M=\bigoplus_{\nu \geq 0} \mathbf{H}^{0}(\mathcal{F}(\nu))$. If $d \geq \operatorname{regularity}(M)$, then by Corollary 2.4 the complex $\mathbf{R}\left(M_{\geq d}\right)$ is acyclic. Thus, if $d>\operatorname{regularity}(M)$, then, since $\mathbf{R}\left(M_{\geq d}\right)$ is minimal, $\operatorname{Hom}_{K}\left(E, M_{d}\right)$ minimally covers the kernel of the map $\operatorname{Hom}_{K}\left(E, M_{d+1}\right) \rightarrow$ $\operatorname{Hom}_{K}\left(E, M_{d+2}\right)$

Fixing $d>$ regularity $(M)$, we may complete $\mathbf{R}\left(M_{\geq d}\right)$ to a minimal Tate resolution $\mathbf{T}(\mathcal{F})$ by adjoining a free resolution of

$$
\operatorname{ker}\left[\operatorname{Hom}_{K}\left(E, M_{d}\right) \rightarrow \operatorname{Hom}_{K}\left(E, M_{d+1}\right)\right] .
$$

Since any two modules representing $\mathcal{F}$ are equal in large degree, the Tate resolution is independent of which $M$ and which large $d$ is chosen, and depends only on the coherent sheaf $\mathcal{F}$. It has the form

$$
\begin{aligned}
\mathbf{T}(\mathcal{F}) & : \cdots \rightarrow T^{d-2} \rightarrow T^{d-1} \rightarrow \operatorname{Hom}_{K}\left(E, \mathbf{H}^{0}(\mathcal{F}(d))\right) \\
& \rightarrow \operatorname{Hom}_{K}\left(E, \mathbf{H}^{0}(\mathcal{F}(d+1))\right) \rightarrow \cdots,
\end{aligned}
$$

where the $T_{i}$ are graded free $E$-modules.

The main theorem of this section expresses the linear part of this Tate resolution in terms of the $S$-modules $\bigoplus_{e} \mathbf{H}^{j}(\mathcal{F}(e))$ given by the (Zariski) cohomology of $\mathcal{F}$. We regard $\bigoplus_{e} \mathbf{H}^{j}(\mathcal{F}(e))$ as a complex of $S$-modules concentrated in cohomological degree $j$. 
Theorem 4.1. If $\mathcal{F}$ is a coherent sheaf on $\mathbf{P}(W)$, then the linear part of the Tate resolution $\mathbf{T}(\mathcal{F})$ is $\bigoplus_{j} \mathbf{R}\left(\bigoplus_{e} \mathbf{H}^{j}(\mathcal{F}(e))\right)$. In particular,

$$
T^{e}=\bigoplus_{j} \operatorname{Hom}_{K}\left(E, \mathbf{H}^{j}(\mathcal{F}(e-j))\right),
$$

where $\mathbf{H}^{j}(\mathcal{F}(e-j))$ is regarded as a vector space of internal degree $e-j$.

A special case of the theorem appears without proof as Remark 3 after Theorem 2 in Bernstein-Gel'fand-Gel'fand [BGG]. The proof below could be extended to cover the case of a bounded complex of coherent sheaves, replacing the cohomology in the formula with hypercohomology. We will postpone the proof of Theorem 4.1 until the end of this section.

Rewriting the indices in Theorem 4.1, we emphasize the fact that we can compute any part of the cohomology of $\mathcal{F}$ from the Tate resolution.

Corollary 4.2. For all $j, \ell \in \mathbf{Z}, \mathbf{H}^{j}(\mathcal{F}(\ell))=\operatorname{Hom}_{E}\left(K, T^{j+\ell}\right)_{-\ell}$.

Corollary 4.2 provides the basis for an algorithm computing the cohomology of $\mathcal{F}$ with any computer program that can provide free resolutions of modules over the symmetric and exterior algebras, such as the program Macaulay2 of Grayson and Stillman [http://www.math.uiuc.edu/Macaulay2/]. For an explanation of the algorithm in practical terms, see Decker and Eisenbud [DE].

To prove Theorem 4.1 we will use the reciprocity result Theorem 3.7 . We actually prove a slightly more general version, involving local cohomology. We write $\mathfrak{m}$ for the homogeneous maximal ideal $S W$ of $S$, and for any graded $S$-module $M$ we write $\mathbf{H}_{\mathfrak{m}}^{j}(M)$ for the $j^{\text {th }}$ local cohomology module of $M$, regarded as a graded $S$-module.

Theorem 4.3. Let $M$ be a graded $S$-module generated in degree d, and having linear free resolution $\mathbf{L}(P)$. Let $\mathbf{F}: \cdots \rightarrow F^{-1} \rightarrow F^{0}$ be the minimal free resolution of $P$. The linear part of $\mathbf{F}$ is

$$
\operatorname{lin}(\mathbf{F})=\bigoplus_{j} \mathbf{R}\left(\mathbf{H}_{\mathfrak{m}}^{j}(M)\right),
$$

where $\mathbf{H}_{\mathfrak{m}}^{j}(M)$ is regarded as a complex with one term, concentrated in cohomological degree $j$. In particular, we have

$$
F^{-i}=\bigoplus_{j} \operatorname{Hom}_{K}\left(E, \mathbf{H}_{\mathfrak{m}}^{j}(M)_{-j-i}\right) .
$$

Proof of Theorem 4.3. We compute the linear part of the free resolution of $P$ by taking the dual (into $K$ ) of the linear part of the injective resolution of $P^{*}$. By Theorem 3.7, the linear part of the injective resolution of $P^{*}$ is $\mathbf{R}\left(\mathbf{H}^{\bullet}\left(\mathbf{L}\left(P^{*}\right)\right)\right)$. It follows at once from the definitions that $\mathbf{L}\left(P^{*}\right)=\operatorname{Hom}_{S}(\mathbf{L}(P), S)$. By Theorem 3.7 once more, $\mathbf{L}(P)$ is the minimal free resolution of $M$, so $\mathbf{H}^{\bullet}\left(\mathbf{L}\left(P^{*}\right)\right)=\operatorname{Ext}_{S}^{\bullet}(M, S)$. Thus the linear part of the free resolution of $P$ is $\left[\operatorname{RExt}_{S}^{\bullet}(M, S)\right]^{*}$, where $\operatorname{Ext}_{S}^{j}(M, S)$ is thought of as a module concentrated in cohomological degree $j$.

Because $E^{*}=\omega_{E}=E \otimes \bigwedge^{v} W$, we have, for any graded vector space $D$, natural identifications

$$
\begin{aligned}
\left(\operatorname{Hom}_{K}(E, D)\right)^{*} & =\left(E^{*} \otimes_{K} D\right)^{*} \\
& =E^{*} \otimes_{K} \bigwedge^{v} W^{*} \otimes_{K} D^{*} \\
& =\operatorname{Hom}_{K}\left(E, D^{*}\right) \otimes_{K} \bigwedge^{v} V .
\end{aligned}
$$


(Here all the duals of $E$-modules are Hom into $K$.) If $D$ has the structure of a graded $S$-module, then $D^{*}$ is again a graded $S$-module, and this becomes an isomorphism of graded $S$-modules. If we think of $D$ as a complex with just one term, in cohomological degree $d$, then $\mathbf{R}(D)^{*}=\mathbf{R}\left(D^{*} \otimes_{K} \bigwedge^{v} V\right)$, where, to make all the indices come out right, we must think of $D^{*} \otimes_{K} \Lambda^{v} V=\left(D \otimes_{K} \Lambda^{v} W\right)^{*}$ as a complex of one term concentrated in cohomological degree $v-d$.

If we take $D=\operatorname{Ext}_{S}^{\ell}(M, S)$, then, by local duality,

$$
\begin{aligned}
D^{*} & =\left(\operatorname{Ext}_{S}^{\ell}(M, S) \otimes_{K} \bigwedge^{v} W \otimes_{K} \bigwedge^{v} V\right)^{*} \\
& =\left(\operatorname{Ext}_{S}^{\ell}\left(M, \omega_{S}\right) \otimes_{K} \bigwedge^{v} V\right)^{*} \\
& =\mathbf{H}_{\mathfrak{m}}^{v-\ell}(M) \otimes \bigwedge^{v} W .
\end{aligned}
$$

Thus

$$
\begin{aligned}
\mathbf{R}\left(\operatorname{Ext}_{S}^{\ell}(M, S)\right)^{*} & =\mathbf{R}\left(\mathbf{H}_{\mathfrak{m}}^{v-\ell}(M) \otimes_{K} \bigwedge^{v} W\right) \otimes \bigwedge^{v} V \\
& =\mathbf{R}\left(\mathbf{H}_{\mathfrak{m}}^{v-\ell}(M)\right),
\end{aligned}
$$

where $\mathbf{H}_{\mathfrak{m}}^{j}(M)$ is regarded as a complex with just one term, of cohomological degree $-j$, as required.

Proof of Theorem 4.1. For each $i=0, \ldots, v-1$ we write $\mathbf{H}^{i}$ for the cohomology module $\bigoplus_{d=-\infty}^{\infty} \mathbf{H}^{i}(\mathcal{F}(d))$. If we choose $d \geq \operatorname{regularity}\left(\mathbf{H}_{\geq 0}^{0}\right)$ as in the construction of $\mathbf{T}(\mathcal{F})$, the module $M:=\mathbf{H}_{\geq d}^{0}$ has a linear free resolution, so we may apply Theorem 4.3. We deduce that the linear part of the free resolution of $P:=$ $\operatorname{ker}\left[\operatorname{Hom}_{K}\left(E, \mathbf{H}^{0}(\mathcal{F}(d))\right) \rightarrow \operatorname{Hom}_{K}\left(\mathbf{H}^{0}(\mathcal{F}(d+1))\right)\right]$ is $\operatorname{lin}(\mathbf{F})=\bigoplus_{j} \mathbf{R}\left(\mathbf{H}_{\mathfrak{m}}^{j}(M)\right)$. If we insist that $d>\operatorname{regularity}\left(\mathbf{H}_{\geq 0}^{0}\right)$, then $\mathbf{H}_{\mathfrak{m}}^{0}(M)=0$. From the exactness of the sequence

$$
0 \rightarrow \mathbf{H}_{\mathfrak{m}}^{0}(M) \rightarrow M \rightarrow \bigoplus_{d=-\infty}^{\infty} \mathbf{H}^{0}(\mathcal{F}(d)) \rightarrow \mathbf{H}_{\mathfrak{m}}^{1}(M) \rightarrow 0
$$

it follows that the local cohomology module $\mathbf{H}_{\mathfrak{m}}^{1}(M)$ ) agrees with the global cohomology module $\mathbf{H}^{0}$ in all degrees strictly less than $d$, and of course we have $\mathbf{H}^{i}=\mathbf{H}_{\mathfrak{m}}^{i+1}(M)$. This concludes the proof.

\section{Powers of the maximal ideal of $E$}

In this section we provide a basic example of the action of the functors $\mathbf{L}$ and $\mathbf{R}$. Among the most interesting graded $S$-modules are the syzygy modules that occur in the Koszul complex. We write

$$
\Omega^{i}=\operatorname{coker}\left[S \otimes_{K} \bigwedge^{i+2} W \rightarrow S \otimes_{K} \bigwedge^{i+1} W\right],
$$

where as usual the elements of $W$ have internal degree 1 , so that the generators of $\Omega^{i}$ have internal degree $i+1$. For example, $\Omega^{-1}=K$ while $\Omega^{0}=(W) \subset S$ and $\Omega^{v-1}=$ $S \otimes \bigwedge^{v} W$, a free module of rank one generated in degree $v$. The sheafifications of these modules are the exterior powers of the cotangent bundle on projective space (see Eisenbud [Eis], Section 17.5, for more details). In this section we shall show that under the functors $\mathbf{L}$ and $\mathbf{R}$ introduced in Section 2 the $\Omega^{i}$ correspond to powers of the maximal ideal $\mathfrak{m} \subset E$. To make the correspondence completely functorial, we make use of the $E$-modules $\mathfrak{m}^{i} \omega_{E}$, where $\omega_{E}=\operatorname{Hom}_{K}(E, K)$. Recall that $\omega_{E}$ is a rank one free $E$-module generated in degree $v$; its generators may be identified with the nonzero elements of $\bigwedge^{v} W$. 
Theorem 5.1. The minimal $S$-free resolution of $\Omega^{i}$ is $\mathbf{L}\left(\omega_{E} / \mathfrak{m}^{v-i} \omega_{E}\right)$; the minimal E-injective resolution of $\omega_{E} / \mathfrak{m}^{v-i} \omega_{E}$ is $\mathbf{R}\left(\Omega^{i}\right)$.

Since $\Omega^{i}$ is generated in degree $i+1$, the complex $\mathbf{R}\left(\Omega^{i}\right)$ begins in cohomological degree $i+1$, and we regard $\omega_{E} / \mathfrak{m}^{v-i} \omega_{E}$ as concentrated in cohomological degree $i+1$.

Proof. The complex $\mathbf{L}\left(\omega_{E}\right)$ is the Koszul complex over $S$, so $\mathbf{L}\left(\omega_{E} / \mathfrak{m}^{v-i} \omega_{E}\right)$ is the truncation

$$
0 \rightarrow S \otimes \bigwedge^{v} W \rightarrow \cdots \rightarrow S \otimes \bigwedge^{i+1} W
$$

which is the resolution of $\Omega^{i}$, proving the first statement. The second statement follows from Theorem 3.7.

Since the $K$-dual of a minimal $E$-injective resolution is a minimal $E$-free resolution, we may immediately derive the free resolution of

$$
\mathfrak{m}^{i+1}=\operatorname{Hom}_{E}\left(\omega_{E} / \mathfrak{m}^{v-i} \omega_{E}, \omega_{E}\right)=\operatorname{Hom}_{K}\left(\omega_{E} / \mathfrak{m}^{v-i} \omega_{E}, K\right) .
$$

Corollary 5.2. The minimal E-free resolution of $\mathfrak{m}^{j}$ is

$$
\operatorname{Hom}_{K}\left(\mathbf{R}\left(\Omega^{j-1}\right), K\right) \text {. }
$$

These resolutions can be made explicit using the Schur functors $\bigwedge_{j}^{i}$ associated to "hook" diagrams (see for example Buchsbaum and Eisenbud [BE] or Akin, Buchsbaum, Weyman $\mathrm{ABW}]$ ). We may define $\bigwedge_{j}^{i}$ (called $L_{j}^{i}$ by Buchsbaum and Eisenbud) by the formula

$$
\bigwedge_{j}^{i}(W)=\operatorname{im}\left[\bigwedge^{i} W \otimes_{K} \operatorname{sym}_{j-1} W \rightarrow \bigwedge^{i-1} W \otimes_{K} \operatorname{sym}_{j} W\right] .
$$

Note that

$$
\bigwedge_{j}^{i}(W)= \begin{cases}0 & \text { if } i<1 \text { or } j<1, \\ \bigwedge^{i} W & \text { if } j=1 \\ \operatorname{Sym}_{j} W & \text { if } i=1 .\end{cases}
$$

Buchsbaum and Eisenbud use these functors to give (among other things) a $G L(W)$ equivariant resolution

$$
\cdots \rightarrow S \otimes_{K} \bigwedge_{j}^{2}(W) \rightarrow S \otimes_{K} \bigwedge_{j}^{1}(W) \rightarrow(W)^{j} \rightarrow 0
$$

of the $j^{\text {th }}$ power $(W)^{j}$ of the maximal ideal of $S$. The $\bigwedge_{j}^{i}$ also provide the terms in the resolutions above:

Corollary 5.3. For $i>0$ the minimal free resolution of $\mathfrak{m}^{i}$ has the form

$$
\cdots \rightarrow E \otimes\left(\bigwedge_{2}^{i} W\right)^{*} \rightarrow E \otimes\left(\bigwedge_{1}^{i} W\right)^{*} \rightarrow \mathfrak{m}^{i} \rightarrow 0 .
$$

For $i<v$ the minimal injective resolution of $\omega_{E} / \mathfrak{m}^{v-i} \omega_{E}$ has the form

$$
\operatorname{Hom}_{K}\left(E, \bigwedge_{1}^{i+1}(W)\right) \rightarrow \operatorname{Hom}_{K}\left(E, \bigwedge_{2}^{i+1}(W)\right) \rightarrow \cdots .
$$

Proof. From the exactness of the Koszul complex we see that $\left(\Omega^{i}\right)_{j}=\bigwedge_{j-i}^{i+1} W$, so the second statement follows from Theorem 5.1. The first statement follows similarly from Corollary 5.2. 
Using the exact sequence

$$
0 \rightarrow \mathfrak{m}^{v-i} \omega_{E} \rightarrow \omega_{E} \rightarrow \omega_{E} / \mathfrak{m}^{v-i} \omega_{E} \rightarrow 0,
$$

we may paste together the injective and free resolutions considered above into the Tate resolution $\mathbf{T}\left(\Omega_{\mathbf{P}}^{i}\right)$.

Corollary 5.4. There is an exact sequence

$$
\begin{aligned}
\mathbf{T}\left(\Omega_{\mathbf{P}}^{i}\right): \cdots & \rightarrow \operatorname{Hom}_{K}\left(E, \bigwedge_{1}^{i+1} W\right) \rightarrow \operatorname{Hom}_{K}\left(E, \bigwedge_{2}^{i+1} W\right) \rightarrow \operatorname{Hom}_{K}(E, K) \\
& \rightarrow \operatorname{Hom}_{K}\left(E,\left(\bigwedge_{1}^{v-i} W\right)^{*}\right) \rightarrow \operatorname{Hom}_{K}\left(E,\left(\bigwedge_{2}^{v-i} W\right)^{*}\right) \rightarrow \cdots,
\end{aligned}
$$

where $\operatorname{Hom}_{K}(E, K)=\omega_{E}$ is the term in cohomological degree $i$.

The following well-known result now follows from Corollary 5.4 by inspection.

Proposition 5.5. In the range $0 \leq j \leq v-1$ or $1 \leq q \leq v-2$,

$$
\mathbf{H}^{q}\left(\mathcal{O}_{\mathbf{P}}(-j) \otimes \Omega_{\mathbf{P}}^{p}(p)\right)= \begin{cases}K, & \text { if } p=q=j, \\ 0, & \text { otherwise }\end{cases}
$$

Proof. Writing the ranks of the free modules in the Tate resolution for $\Omega_{\mathbf{P}}^{p}$ in Macaulay notation, we find

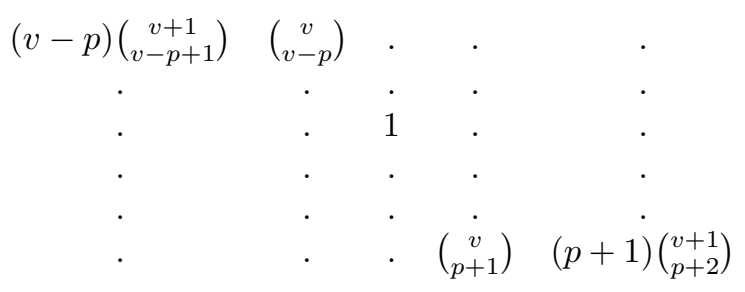

with the rank 1 module sitting in homological degree $p$ and the ingoing and outgoing map from it given by bases of the forms in $\bigwedge^{v-p} V$ and $\bigwedge^{p+1} V$ respectively.

If we shift the rank 1 module into homological degree 0 , then we have the Tate resolution of $\Omega^{p}(p)$. Following Beilinson ([Bei], Lemma 2), we can also compute $\operatorname{Hom}\left(\Omega^{i}(i), \Omega^{j}(j)\right)$ for any $i, j$, which will play a major role in Section 6 .

Proposition 5.6. If $\Omega^{i}(i)$ are the $S$-modules defined in Section 5 and $0 \leq i, j<v$, then

$$
\operatorname{hom}_{S}\left(\Omega^{i}(i), \Omega^{j}(j)\right)=\bigwedge^{i-j} V=\operatorname{hom}_{E}\left(\omega_{E}(i), \omega_{E}(j)\right)
$$

where in each case hom denotes the (degree 0) homomorphisms; for other values of $i, j$ the left-hand side is 0 . The product of homomorphisms corresponds to the product in $\bigwedge V$.

Proof. The modules $\Omega^{i}(i)$ are 0 for $i<0$ and $i \geq v$. For $0 \leq i<v$ they have linear resolution, so we may apply Theorem 3.7. As they are 0 in degrees $<1$ and generated in degree 1 , we have $\mathbf{H}^{1} \mathbf{R}\left(\Omega^{i}(i)\right)=\omega_{E}(i) / \mathfrak{m}^{v-i} \omega_{E}(i)$ if $v>i$, by Theorem 5.1. For $0 \leq i, j<v$, maps $\omega_{E}(i) / \mathfrak{m}^{v-i} \omega_{E}(i) \rightarrow \omega_{E}(j) / \mathfrak{m}^{v-j} \omega_{E}(j)$ are in one-to-one correspondence with maps $\omega_{E}(i) \rightarrow \omega_{E}(j)$. Since $\omega_{E}$ is a rank one free $E$-module, these may be identified with elements of $E_{j-i}=\bigwedge^{i-j} V$. 


\section{BEILINSON'S MONAD}

Beilinson's paper [Bei] contains two main results. The first says that, given a sheaf $\mathcal{F}$ on a projective space $\mathbf{P}=\mathbf{P}(W)$, there is a complex

$$
\mathcal{B}: \cdots \rightarrow B^{-1} \rightarrow B^{0} \rightarrow B^{1} \rightarrow \cdots
$$

with

$$
B^{e}=\bigoplus_{j} \mathbf{H}^{j}(\mathcal{F}(e-j)) \otimes \Omega_{\mathbf{P}}^{j-e}(j-e)
$$

such that $\mathcal{B}$ is exact except at $B^{0}$ and the homology at $B^{0}$ is $\mathcal{F}$.

We show that the complex $\mathcal{B}$ may be obtained by applying a certain functor to the Tate resolution $\mathbf{T}(\mathcal{F})$ over $E$. Beilinson's second main result gives another monad, which we will treat in Theorem 8.1.

Given any graded free complex $\mathbf{T}$ over $E$, we may write each module of $\mathbf{T}$ as a direct sum of copies of $\omega_{E}(i)=\operatorname{Hom}_{K}(E, K(i))$ with varying $i$. We define $\Omega(\mathbf{T})$ to be the complex of sheaves on $\mathbf{P}$ obtained by replacing each summand $\omega_{E}(i)$ by the sheaf $\Omega_{\mathbf{P}}^{i}(i)$ and using the isomorphism of Hom in Proposition 5.6 to provide the maps.

Theorem 6.1. If $\mathcal{F}$ is a coherent sheaf on $\mathbf{P}(W)$ with associated Tate resolution $\mathbf{T}(\mathcal{F})$, then the only homology of $\Omega(\mathbf{T}(\mathcal{F})$ ) is in cohomological degree 0 , and is isomorphic to $\mathcal{F}$.

Proof. To simplify the notation we set $\mathbf{T}=\mathbf{T}(\mathcal{F})$, and we let $\overline{\mathbf{T}}$ be $\mathbf{T}$ modulo the elements of internal degree $\geq 0$. Let $\mathcal{L}$ be the double complex of sheaves that arises by sheafifying the double complex of $S$-modules used to construct the complex $\mathbf{L}(\mathbf{T})$; that is, if $T^{e}$ is the component of $\mathbf{T}$ of cohomological degree $e$, and $T_{j}^{e}$ is its component of internal degree $j$, then the double complex $\mathcal{L}$ has the form

$\mathcal{L}:$

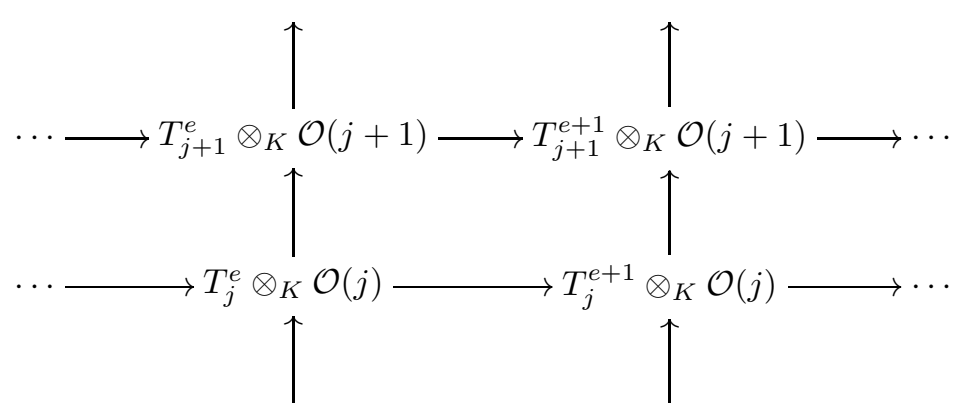

Since $\mathbf{T}$ is exact, the rows are exact; since the columns are direct sums of sheafified Koszul complexes over $S$, they are exact as well.

Choose an integer $f>>0$ (greater than the regularity of $\mathcal{F}$ will be sufficient) and let $\mathcal{L}^{\prime}$ be the double complex obtained from $\mathcal{L}$ by taking only those terms $T_{j}^{e} \otimes_{K} \mathcal{O}(j)$ with $e<f$ and $j>0$. If $e<<0$, then $T^{e}$ is generated in negative degrees, so the double complex $\mathcal{L}^{\prime}$ is finite, and is exact except at the right $(e=f-1)$ and at $j=1$. An easy spectral sequence argument shows that the complex obtained as the vertical homology of $\mathcal{L}^{\prime}$ has the same homology as the complex obtained as the horizontal homology of $\mathcal{L}^{\prime}$.

If we write $T^{e}$ as a sum of copies of $\omega_{E}(i)$ for various $i$, then the $e^{\text {th }}$ column of $\mathcal{L}^{\prime}$ is correspondingly a sum of copies of the sheafification of $\mathbf{L}\left(\omega_{E}(i) / \mathfrak{m}^{v-i} \omega_{E}(i)\right)$. 
As in Theorem 5.1, the vertical homology of this column is correspondingly a sum of copies of $\Omega_{\mathbf{P}}^{i}(i)$; that is, it is $\Omega\left(T^{e}\right)$. Thus the complex obtained as the vertical homology of $\mathcal{L}^{\prime}$ is $\Omega(\mathbf{T})$.

As $e$ goes to infinity, the degrees of the generators of $T^{e}$ become more and more positive; thus for $e$ large the $e^{\text {th }}$ column of $\mathcal{L}^{\prime}$ is the same as that of $\mathcal{L}$, that is, it is $\mathbf{L}\left(T^{e}\right)$. Since $f>>0$, the horizontal homology of $\mathcal{L}^{\prime}$ is the sheafification of $\mathbf{L}(H)$, where $H$ is the homology of $\mathbf{T}_{<f}$. As $\mathbf{T}$ is exact, $H$ may also be written as the homology of $\mathbf{T}_{\geq f}$. Taking $f>$ regularity $\mathcal{F}$ and using Theorem 3.7, we see that $\mathbf{L}(H)$ is a free resolution of the module $\bigoplus_{e \geq f} \mathbf{H}^{0}(\mathcal{F}(e))$, whose sheafification is $\mathcal{F}$, as required.

Corollary 6.2. The map in the complex $\Omega(\mathbf{T}(\mathcal{F}))$ corresponding to

$$
\mathbf{H}^{j}(\mathcal{F}(j-i)) \otimes \Omega_{\mathbf{P}}^{i-j}(i-j) \longrightarrow \mathbf{H}^{j}(\mathcal{F}(j-i+1)) \otimes \Omega_{\mathbf{P}}^{i-j-1}(i-j-1)
$$

corresponds to the multiplication map $W \otimes_{K} \mathbf{H}^{j}(\mathcal{F}(j-i)) \longrightarrow \mathbf{H}^{j}(\mathcal{F}(j-i+1))$.

Proof. This follows from Theorem 4.1, since we have identified not only the modules but the maps in the linear strands of the resolution.

Corollary 6.3. The maps in the complex $\Omega(\mathbf{T}(\mathcal{F}))$ correspond to the maps in the complex $\Omega(\mathbf{T}(\mathcal{F}(1)))$ under the natural correspondence

$$
\operatorname{hom}_{\mathbf{P}}\left(\Omega_{\mathbf{P}}^{i}(i), \Omega_{\mathbf{P}}^{j}(j)\right)=\bigwedge^{i-j} V=\operatorname{hom}_{\mathbf{P}}\left(\Omega_{\mathbf{P}}^{i+1}(i+1), \Omega_{\mathbf{P}}^{j+1}(j+1)\right)
$$

whenever $0 \leq i, i+1, j, j+1<v$.

Proof. The Tate resolution $\mathbf{T}(\mathcal{F}(1))$ is obtained by simply shifting $\mathbf{T}(\mathcal{F})$.

\section{EXAMPLES}

Example 7.1. Let $C$ be an elliptic quartic curve in $\mathbf{P}^{3}$, and consider $\mathcal{O}_{C}$ as a sheaf on $\mathbf{P}^{3}$. Write $\omega_{E}=\bigwedge^{v} W \otimes E$, as usual. Computing cohomology, one sees that $\mathbf{T}\left(\mathcal{O}_{C}\right)$ has the form

$$
\cdots \longrightarrow \omega_{E}^{8}(2) \longrightarrow \omega_{E} \oplus \omega_{E}^{4}(1) \longrightarrow{ }^{d} \omega_{E}^{4}(-1) \oplus \omega_{E} \longrightarrow \omega_{E}^{8}(-2) \longrightarrow \cdots .
$$

If $C \subset \mathbf{P}^{3}$ is taken to be Heisenberg invariant, say $C=\left\{x_{0}^{2}+x_{2}^{2}+\lambda x_{1} x_{3}=\right.$ $\left.x_{1}^{2}+x_{3}^{2}+\lambda x_{0} x_{2}=0\right\}$ for some $\lambda \in \mathbf{A}_{k}^{1}$, then $d$ can be represented by the matrix

$$
\left(\begin{array}{ccccc}
0 & e_{0} & e_{1} & e_{2} & e_{3} \\
e_{0} & -\lambda e_{1} e_{3} & e_{2} e_{3} & 0 & e_{1} e_{2}+\frac{\lambda^{2}}{2} e_{0} e_{3} \\
e_{1} & e_{2} e_{3} & \lambda e_{0} e_{2} & -e_{0} e_{3}-\frac{\lambda^{2}}{2} e_{1} e_{2} & 0 \\
e_{2} & 0 & -e_{0} e_{3}-\frac{\lambda^{2}}{2} e_{1} e_{2} & \lambda e_{1} e_{3} & e_{0} e_{1} \\
e_{3} & e_{1} e_{2}+\frac{\lambda^{2}}{2} e_{0} e_{3} & 0 & e_{0} e_{1} & -\lambda e_{0} e_{2}
\end{array}\right) .
$$

Example 7.2. The rational normal curve. Let $C \subset \mathbf{P}^{d}$ be the curve parametrized by $(s: t) \mapsto\left(s^{d}: s^{d-1} t: \ldots: t^{d}\right)$. We consider the line bundles $\mathcal{L}_{k}$ on $C$ associated to $\bigoplus_{m=0}^{\infty} H^{0}\left(\mathbf{P}^{1}, \mathcal{O}(k+m d)\right)$ for $k=-1, \ldots, d-2$. The Tate resolution $\mathbf{T}\left(\mathcal{L}_{k}\right)$ has Betti numbers

$$
\begin{aligned}
& \text { ** } 3 d-k-1 \quad 2 d-k-1 \quad d-k-1 \quad . \quad \ldots
\end{aligned}
$$

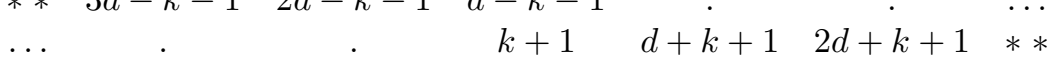


The $[(k+1)+(d-k-1)] \times(2 d-k-1)$ matrix near the middle and the matrices surrounding it have in case $d=4$ and $k=1$ the following shapes:

$$
\left(\begin{array}{cccc}
0 & e_{0} & e_{0} e_{2} & e_{0} e_{1} \\
e_{0} & e_{1} & e_{1} e_{2}+e_{0} e_{3} & e_{0} e_{2} \\
e_{1} & e_{2} & e_{1} e_{3}+e_{0} e_{4} & e_{0} e_{3} \\
e_{2} & e_{3} & e_{1} e_{4} & e_{0} e_{4} \\
e_{3} & e_{4} & 0 & 0 \\
e_{4} & 0 & 0 & 0
\end{array}\right),\left(\begin{array}{cccccc}
0 & 0 & e_{0} e_{4} & e_{1} e_{4} & e_{2} e_{4} & e_{3} e_{4} \\
0 & 0 & e_{0} e_{3} & e_{1} e_{3}+e_{0} e_{4} & e_{2} e_{3}+e_{1} e_{4} & e_{2} e_{4} \\
0 & e_{0} & e_{1} & e_{2} & e_{3} & e_{4} \\
e_{0} & e_{1} & e_{2} & e_{3} & e_{4} & 0
\end{array}\right)
$$

and

$$
\left(\begin{array}{cccccccccc}
e_{0} & e_{1} & e_{2} & e_{3} & e_{4} & 0 & 0 & 0 & 0 & 0 \\
0 & e_{0} & e_{1} & e_{2} & e_{3} & e_{4} & 0 & 0 & 0 & 0 \\
0 & 0 & e_{0} & e_{1} & e_{2} & e_{3} & e_{4} & 0 & 0 & 0 \\
0 & 0 & 0 & e_{0} & e_{1} & e_{2} & e_{3} & e_{4} & 0 & 0 \\
0 & 0 & 0 & 0 & e_{0} & e_{1} & e_{2} & e_{3} & e_{4} & 0 \\
0 & 0 & 0 & 0 & 0 & e_{0} & e_{1} & e_{2} & e_{3} & e_{4}
\end{array}\right)
$$

All other matrices look similar to the last one.

In case $k=-1$ we obtain a $d \times d$ symmetric matrix of 2 -forms:

$$
\left(\begin{array}{cccc}
e_{0} e_{1} & e_{0} e_{2} & e_{0} e_{3} & e_{0} e_{4} \\
e_{0} e_{2} & e_{1} e_{2}+e_{0} e_{3} & e_{1} e_{3}+e_{0} e_{4} & e_{1} e_{4} \\
e_{0} e_{3} & e_{1} e_{3}+e_{0} e_{4} & e_{2} e_{3}+e_{1} e_{4} & e_{2} e_{4} \\
e_{0} e_{4} & e_{1} e_{4} & e_{2} e_{4} & e_{3} e_{4}
\end{array}\right)
$$

If we interpret 2 -forms as coordinate functions

$$
e_{i j}=e_{i} e_{j}=e_{i} \wedge e_{j} \in \mathbf{H}^{0}(\mathbf{G}(W, 2), \mathcal{O}(1)) \cong \mathbf{H}^{0}\left(\mathbf{P}\left(\bigwedge^{2} V\right), / \mathcal{O}(1)\right)
$$

on the Grassmannian of codimension 2 linear subspaces in $\mathbf{P}(W)$, then the determinant of the matrix above defines the Chow divisor of $C \subset \mathbf{P}^{d}$, which is by definition the hypersurface $\left\{\mathbf{P}^{d-2} \in \mathbf{G}(W, 2) \mid \mathbf{P}^{d-2} \cap C \neq \emptyset\right\}$. Eisenbud and Schreyer [ES1] give a general computation of Chow forms along these lines.

Example 7.3. The Horrocks-Mumford bundle in $\mathbf{P}^{4}$. A famous Beilinson monad was discovered by Horrocks and Mumford [HM]: Consider for $\mathbf{P}^{4}$ the Tate resolution $\mathbf{T}(\varphi)$ of the matrix

$$
\varphi=\left(\begin{array}{ccccc}
e_{1} e_{4} & e_{2} e_{0} & e_{3} e_{1} & e_{4} e_{2} & e_{0} e_{3} \\
e_{2} e_{3} & e_{3} e_{4} & e_{4} e_{0} & e_{0} e_{1} & e_{1} e_{2}
\end{array}\right) .
$$

By direct computation we find the Betti numbers

$$
\begin{array}{ccccccccccccc}
? & . & . & . & . & . & . & . & . & . & . & . & \ldots \\
* * & 100 & 35 & 4 & 0 & . & . & . & . & . & . & . & \ldots \\
\ldots & 0 & 2 & 10 & 10 & 5 & 0 & . & . & . & . & . & \ldots \\
\ldots & . & . & . & . & 0 & 2 & 0 & . & . & . & . & \ldots \\
\ldots & . & . & . & . & . & 0 & 5 & 10 & 10 & 2 & 0 & \ldots \\
\ldots & . & . & . & . & . & . & . & 0 & 4 & 35 & 100 & * *
\end{array}
$$

To deduce that this Tate resolution comes from a sheaf, we use

Lemma 7.4. Let $\mathbf{T}$ be a Tate resolution over $E$. Suppose that $\left(K \otimes_{E} T^{0}\right)_{j}=0$ for all $j<0$. Then $\left(K \otimes_{E} T^{l}\right)_{m}=0$ if $l>0$ and $m<l$, or if $l<0$ and $m<l-n$. 
Proof. Pictorially the statement says that vanishing in a single $T^{e}$ implies vanishing in the indicated range:

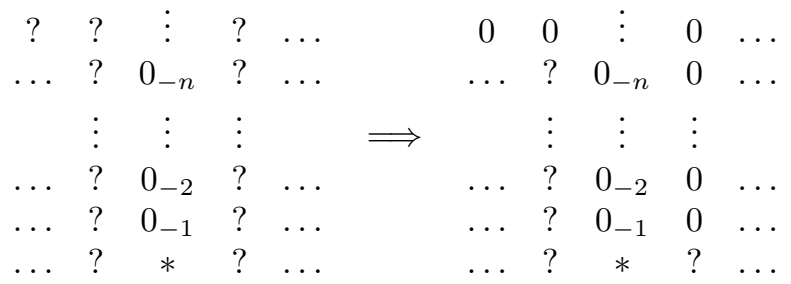

The first vanishing follows, because $\operatorname{Hom}_{E}(\mathbf{T}, E)$ is also a minimal complex. For the second we note for $P=\operatorname{ker}\left(T^{0} \rightarrow T^{1}\right)$ that $P_{j}=0$ holds for all $j<-v$ by our assumption. By Corollary 5.3, $\left(K \otimes_{E} T^{l}\right)=\operatorname{Tor}_{-l-1}^{E}(K, P)$, which is a subquotient of $\left(\operatorname{Sym}_{-l-1} W\right)^{*} \otimes_{K} P$. Thus this group vanishes in all degrees $m<l-v+1$.

Example 7.3, continued. By applying Lemma 7.4 to a shift of $\mathbf{T}(\varphi)$ and $\operatorname{Hom}(\mathbf{T}(\varphi), E)$ we see that the $\mathbf{T}(\varphi)$ has terms only in the indicated range of rows; in particular, the rows with the question marks contain only zeros. So $\mathbf{T}(\varphi)$ is the Tate resolution of some sheaf $\mathcal{F}$. Moreover $\mathcal{F}$ is a bundle, since the middle cohomology has only finitely many terms. The $4^{\text {th }}$ difference function of $\chi(\mathcal{F}(m))$ has constant value 2. So $\mathcal{F}$ has rank 2. It is the famous bundle on $\mathbf{P}^{4}$ discovered by Horrocks and Mumford [HM]. In Decker and Schreyer [DS1] it is proved that any stable rank 2 vector bundle on $\mathbf{P}^{4}$ with the same Chern classes equals $\mathcal{F}$ up to projectivities.

\section{FREE MONADS}

A free monad $\mathcal{L}$ for a coherent sheaf $\mathcal{F}$ is a finite complex

$$
0 \rightarrow \mathcal{L}^{-N} \rightarrow \cdots \rightarrow \mathcal{L}^{-1} \rightarrow \mathcal{L}^{0} \rightarrow \mathcal{L}^{1} \rightarrow \cdots \rightarrow \mathcal{L}^{M} \rightarrow 0
$$

on $\mathbf{P}^{n}=\mathbf{P}(W)$ whose components $\mathcal{L}^{i}$ are direct sums of line bundles and whose homology is $\mathcal{F}$ :

$$
\mathbf{H}^{\bullet}(\mathcal{L})=\mathbf{H}^{0}(\mathcal{L}) \cong \mathcal{F} .
$$

The complex of twisted global sections of $\mathcal{L}$ is a complex $L=\Gamma_{*}(\mathcal{L})$ of free $S$ modules. If $L$ is a minimal complex, then we speak of a minimal free monad. The most familiar free monads are the sheafifications of the minimal free resolutions of the modules $\bigoplus_{m \geq m_{0}} \mathbf{H}^{0} \mathcal{F}(m)$ for various $m_{0}$.

Free monads have been constructed by Horrocks [Hor], Barth [Bar], Bernstein, Gel'fand and Gel'fand BGG and Beilinson Bei], mainly for the study of vector bundles on projective spaces. Rao [Rao and Martin-Deschamps and Perrin [MP used free monads in their studies of space curves. Fløystad [Flo3] gave a complete classification of a certain class of linear monads on projective spaces. The general construction of free monads is the following:

Theorem 8.1. Let $\mathcal{F}$ be a coherent sheaf on $\mathbf{P}^{n}$, and let $\mathbf{T}^{\prime}$ be a left-bounded complex of finite free $E$-modules with $\mathbf{T}^{\prime \geq r}=\mathbf{T}(\mathcal{F})^{\geq r}$ for some $r$. Let $L=\min \mathbf{L}\left(\mathbf{T}^{\prime}\right)$ be the minimalized complex of the BGG transform $\mathbf{L}\left(\mathbf{T}^{\prime}\right)$. Its sheafication $\mathcal{L}\left(\mathbf{T}^{\prime}\right)=\tilde{L}$ is a free monad for $\mathcal{F}$. Every minimal free monad $\mathcal{L}$ of $\mathcal{F}$ arises as $\mathcal{L}=\mathcal{L}\left(\mathbf{T}^{\prime}\right)$ in this way with $\mathbf{T}^{\prime}=\min \mathbf{R}(L)$. 
Proof. Suppose $\mathbf{T}^{\prime}$ satisfies the assumption. Since $\mathbf{T}^{\prime}$ is left-bounded and acyclic for large degrees, $L=\min \mathbf{L}\left(\mathbf{T}^{\prime}\right)$ is a finite complex by the second statement in Corollary 3.6. The cohomology of the complex $L$ can be computed by taking linear parts: $\bigoplus_{i} \mathbf{R}\left(\mathbf{H}^{i}(L)\right)=\operatorname{lin}(\mathbf{R}(L))=\operatorname{lin}\left(\mathbf{R}\left(\mathbf{L}\left(\mathbf{T}^{\prime}\right)\right)\right)=\operatorname{lin}\left(\mathbf{T}^{\prime}\right)$ by Corollary 3.6. So $\mathbf{H}^{j}(L)$ is of finite length for $j \neq 0$, and sheafifying gives

$$
\mathbf{H}^{\bullet}(\mathcal{L})=\mathbf{H}^{0}(\mathcal{L})=\left(\Gamma_{\geq r} \mathcal{F}\right)=\mathcal{F} .
$$

Conversely, if $\mathcal{L}$ is a free monad for $\mathcal{F}$ and $L=\Gamma_{*} \mathcal{L}$, then $\mathbf{H}^{j}(L)$ has finite length for $j \neq 0$. Thus $\mathbf{T}^{\prime}=\min \mathbf{R}(L)$ is a left-bounded complex with $\mathbf{T}^{\prime} \geq r=\mathbf{T}(\mathcal{F}) \geq r$ by Corollary 3.6, and $\min \mathbf{L}\left(\mathbf{T}^{\prime}\right)=\min \mathbf{L}(\min \mathbf{R}(L))=\min \mathbf{L R}(L)=\min L=L$.

Example 8.2. Consider $\mathcal{F}=\mathcal{O}_{p}$, the structure sheaf of a point in $\mathbf{P}^{1}$. Its Tate resolution is periodic:

$$
\text { ‥e } \stackrel{e}{\longrightarrow} \omega_{E}(1) \stackrel{e}{\longrightarrow} \omega_{E} \stackrel{e}{\longrightarrow} \omega_{E}(-1) \stackrel{e}{\longrightarrow} \cdots .
$$

If we take $\mathbf{T}^{\prime}$ to be the truncation

$$
0 \longrightarrow \omega_{E} \stackrel{e}{\longrightarrow} \omega_{E}(-1) \stackrel{e}{\longrightarrow} \cdots,
$$

then the monad $\mathcal{L}\left(\mathbf{T}^{\prime}\right)$ is the sheafified free resolution

$$
0 \longrightarrow \mathcal{O}(-1) \longrightarrow^{x} \mathcal{O} \longrightarrow 0 \text {. }
$$

If instead we take $\mathbf{T}^{\prime}$ to be the complex

$$
0 \longrightarrow \omega_{E} \longrightarrow \text { ef } \omega_{E}(-2) \stackrel{e}{\longrightarrow} \omega_{E}(-3) \stackrel{e}{\longrightarrow} \cdots,
$$

then $L\left(\mathbf{T}^{\prime}\right)$ is the free resolution of $S /\left(x^{2}, x y\right)$, which has sheafification $\mathcal{L}\left(\mathbf{T}^{\prime}\right)$ of the form

$$
0 \longrightarrow \mathcal{O}(-3) \longrightarrow \mathcal{O}(-2)^{2} \longrightarrow \mathcal{O} \longrightarrow 0 \text {. }
$$

For the rest of this section we will study a class of free monads we call partition monads (because they partition the cohomology of $\mathcal{F}$ into two simple pieces, which occur as $\mathbf{H}^{\bullet}(L)$ and $\left.\mathbf{H}^{\bullet}\left(L^{*}\right)\right)$. This class includes the sheafified free resolutions and most of the other free monads found in the literature.

Definition 8.3. Partition monads. Given a weakly increasing sequence of integers

$$
\mu=\left(m_{0} \leq m_{1} \leq \cdots \leq m_{n}\right),
$$

we define $\mathbf{T}_{\mu}(\mathcal{F})$ to be the subcomplex of $\mathbf{T}(\mathcal{F})$ given by

$$
T_{\mu}^{e}(\mathcal{F})=\bigoplus_{i: e \geq m_{i}} \mathbf{H}^{i} \mathcal{F}(e-i) \otimes_{K} \omega_{E}(i-e) .
$$

We shall also make use of the complementary complex $\mathbf{T}^{\mu}$ defined by the exact sequence

$$
0 \rightarrow \mathbf{T}_{\mu} \rightarrow \mathbf{T} \rightarrow \mathbf{T}^{\mu} \rightarrow 0 .
$$

We set $L_{\mu}(\mathcal{F}):=\min \mathbf{L}\left(\mathbf{T}_{\mu}(\mathcal{F})\right)$, and write $\mathcal{L}_{\mu}(\mathcal{F})=\tilde{L}_{\mu}(\mathcal{F})$ for the monad which is its sheafification.

Example 8.4. Free resolutions. Let $m_{0}$ be any integer, and choose $m_{1}, \ldots, m_{n}$ greater than the Castelnuovo-Mumford regularity of $\mathcal{F}$. The complex $\mathbf{T}_{\mu}(\mathcal{F})$ is $\mathbf{R}\left(\bigoplus_{m \geq m_{0}} \mathbf{H}^{0}(\mathcal{F}(m))\right)$. Thus by Theorem 2.6 the complex $L_{\mu}(\mathcal{F})$ is the minimal free resolution of $\bigoplus_{m \geq m_{0}} \mathbf{H}^{0}(\mathcal{F}(m))$. 
Example 8.5. Linear monads. Consider the case $m=m_{0}=m_{1}=\cdots=m_{n}$. In this case $\mathbf{T}_{\mu}=\mathbf{T}^{\geq m}$ is an injective resolution of $P_{m}=\operatorname{ker}\left(T^{m} \rightarrow T^{m+1}\right)$, and $\mathcal{L}_{\mu}=\widetilde{\mathbf{L}}\left(P_{m}\right)$ has only linear maps.

Like free resolutions, partition monads enjoy a strong homotopy functoriality:

Proposition 8.6. The partition monad $\mathcal{L}_{\mu}(\mathcal{F})$ is functorial in $\mathcal{F}$ up to homotopy of complexes in such a way that if $\phi: \mathcal{F} \rightarrow \mathcal{G}$ is a map, then $\phi=\mathbf{H}^{0} \mathcal{L}_{\mu}(\phi)$. Moreover, any map of complexes $\mathcal{L}_{\mu}(\mathcal{F}) \rightarrow \mathcal{L}_{\mu}(\mathcal{G})$ is determined up to homotopy by the induced map $\mathcal{F}=\mathbf{H}^{0}\left(\mathcal{L}_{\mu}(\mathcal{F})\right) \rightarrow \mathbf{H}^{0}\left(\mathcal{L}_{\mu}(\mathcal{G})\right)=\mathcal{G}$.

Proof. The first statement follows from the homotopy functoriality of $\mathbf{T}$ and $\mathbf{L}$. For the second statement, it suffices to show that every map $\mathcal{L}_{\mu}(\mathcal{F}) \rightarrow \mathcal{L}_{\mu}(\mathcal{G})$ is homotopic to a map of the form $\mathcal{L}_{\mu}(\phi)$. But every map $\mathbf{T}_{\mu}(\mathcal{F}) \rightarrow \mathbf{T}_{\mu}(\mathcal{G})$ is homotopic to a map $\mathbf{T}_{\mu}(\phi)$, and since $\mathbf{T}_{\mu}(\mathcal{G})$ is an injective resolution, it is homotopic to $\mathbf{R L T}_{\mu}(\mathcal{G})$. Using the adjointness of $\mathbf{R}$ and $\mathbf{L}$, we see that, up to homotopy, indeed every map is in the image of the the composite homomorphism

$$
\begin{aligned}
\operatorname{Hom}(\mathcal{F}, \mathcal{G}) & \rightarrow \operatorname{Hom}\left(\mathbf{T}_{\mu}(\mathcal{F}), \mathbf{T}_{\mu}(\mathcal{G})\right) \\
& \rightarrow \operatorname{Hom}\left(\mathbf{T}_{\mu}(\mathcal{F}), \mathbf{R L T}_{\mu}(\mathcal{G})\right) \\
& =\operatorname{Hom}\left(\mathbf{L} \mathbf{T}_{m} u(\mathcal{F}), \mathbf{L} \mathbf{T}_{\mu}(\mathcal{G})\right) \\
& \rightarrow \operatorname{Hom}\left(\mathcal{L}_{\mu}(\mathcal{F}), \mathcal{L}_{\mu}(\mathcal{G})\right)
\end{aligned}
$$

Proposition 8.7. The cohomology of the complexes $L_{\mu}$ and $L_{\mu}^{*}=\operatorname{Hom}_{S}\left(L_{\mu}, S\right)$ are given by

$$
\mathbf{H}^{i} L_{\mu}=\bigoplus_{d \geq m_{i}-i} \mathbf{H}^{i} \mathcal{F}(d) ; \quad \mathbf{H}^{n-i} L_{\mu}^{*}=\bigoplus_{d<m_{i}-i} \mathbf{H}^{i}(\mathcal{F}(d))^{*} \otimes \bigwedge^{v} V,
$$

where $\mathbf{H}^{i} \mathcal{F}(d)$ occurs in degree $d$ and $\mathbf{H}^{i}(\mathcal{F}(d))^{*} \otimes \bigwedge^{v} V$ occurs in degree $-n-d-1$. In particular, for $j<0$ we have $\mathbf{H}^{j} L_{\mu}=\mathbf{H}^{j} L_{\mu}^{*}=0$.

Proof. Let $\mathbf{P}_{\mu}$ be the complex

$$
0 \rightarrow T_{\mu}^{m_{0}} \rightarrow \cdots \rightarrow T_{\mu}^{m_{n}} \rightarrow \operatorname{im}\left(T_{\mu}^{m_{n}} \rightarrow T_{\mu}^{m_{n}+1}\right) \rightarrow 0,
$$

so that the complex $\mathbf{T}_{\mu}$ is an injective resolution of $\mathbf{P}_{\mu}$. By part $b$ ) of Theorem 3.7 the linear part of the injective resolution of $\mathbf{P}_{\mu}$ is the sum of the linear complexes $\mathbf{R}\left(\mathbf{H}^{i} \mathbf{L}\left(\mathbf{P}_{\mu}\right)\right)$. Thus $\mathbf{H}^{i} L_{\mu}=\mathbf{H}^{i} \mathbf{L}\left(\mathbf{P}_{\mu}\right)=\bigoplus_{e \geq m_{i}} \mathbf{H}^{i} \mathcal{F}(e-i)$, by the definition of $\mathbf{T}_{\mu}$.

For the proof of the second formula we first observe that $L_{\mu}^{*}=\operatorname{Hom}_{S}\left(L_{\mu}, S\right)=$ $\min \mathbf{L}\left(\operatorname{Hom}_{K}\left(\mathbf{P}_{\mu}, K\right)\right)$. Since $\mathbf{T}(\mathcal{F})$ is exact, the induced map $\mathbf{T}^{\mu}[-1] \rightarrow \mathbf{T}_{\mu}$ is a quasi-isomorphism. Moreover, this map factors through $\mathbf{P}_{\mu}$. Thus $\mathbf{T}^{\mu}[-1]$ is a projective resolution of $\mathbf{P}_{\mu}$, and $\operatorname{Hom}_{K}\left(\mathbf{T}^{\mu}, K\right)[1]$ is an injective resolution of $\operatorname{Hom}_{K}\left(\mathbf{P}_{\mu}, K\right)$. The terms with $\mathbf{H}^{i}$ on the right-hand side of the desired equality correspond to the $(v-1-i)^{\text {th }}$ linear strand of $\operatorname{Hom}\left(\mathbf{T}^{\mu}, K\right)[1]$. Again by Theorem 3.7 , the second formula follows.

Corollary 8.8. Any partition monad $\mathcal{L}_{\mu}(\mathcal{F})$ satisfies $\mathcal{L}_{\mu}^{i}=0$ for $|i|>n$.

Proof. If $L_{\mu}^{i} \neq 0$ but $L_{\mu}^{i+1}=0$, then Nakayama's Lemma implies that $\mathbf{H}^{i}\left(L_{\mu}\right) \neq 0$, and similarly for the dual. Proposition 8.7 completes the argument. 
It is easy to give bounds on the line bundles that can occur in a partition monad. Given the sequence $\mu=\left(m_{0} \leq \cdots \leq m_{n}\right)$, it will be convenient to extend the definition of $m_{i}$ to all $i \in \mathbf{Z}$ by the formulas

$$
m_{i}= \begin{cases}m_{0} & \text { if } i<0, \\ m_{n} & \text { if } i>n .\end{cases}
$$

Corollary 8.9. If $\mathcal{O}(-a)$ is a summand of the $i^{\text {th }}$ term of the partition monad $\mathcal{L}_{\mu}(\mathcal{F})$, then

$$
m_{i} \leq a+i \leq m_{i+n},
$$

where the definition of $m_{i}$ is extended to all $i \in \mathbf{Z}$ as above.

Proof. By Corollary 3.6

$$
\operatorname{lin} L_{\mu}=\operatorname{lin} \mathbf{L}\left(\mathbf{T}_{\mu}(\mathcal{F})\right)=\bigoplus_{e} \mathbf{L}\left(\mathbf{H}^{e} \mathbf{T}_{\mu}(\mathcal{F})\right),
$$

so the $i^{\text {th }}$ term of $L_{\mu}$ is $\bigoplus_{e} \mathbf{H}^{e}\left(\mathbf{T}_{\mu}\right)_{e-i} \otimes S(i-e)$. For the first inequality we have to show that if $\mathbf{H}^{e}\left(\mathbf{T}_{\mu}\right)_{e-i} \neq 0$, then $m_{i} \leq-(i-e)+i=e$. Since

$$
T_{\mu}^{e}=\bigoplus_{j: e \geq m_{j}} \omega_{E}(j-e) \otimes \mathbf{H}^{j} \mathcal{F}(e-j)
$$

and $\omega_{E}$ is zero in negative degrees, the condition $\left(T_{\mu}^{e}\right)_{e-i} \neq 0$ implies $j-e+e-i \geq 0$ for some $j$ with $e \geq m_{j}$. Thus $i \leq j$ and $m_{i} \leq m_{j} \leq e$, as desired.

For the second inequality we argue similarly, using $\mathbf{H}^{e}\left(\mathbf{T}_{\mu}\right) \cong \mathbf{H}^{e-1}\left(\mathbf{T}^{\mu}\right)$.

Note that if $\mathcal{L}$ is a monad for a sheaf $\mathcal{F}$, then so is $\mathcal{L} \oplus \mathcal{A}$, where $\mathcal{A}$ is an acyclic complex - for example, the sheafification of the free resolution of a module of finite length.

The main result of this section is that partition monads are characterized by the conditions in Corollary 8.8 and Corollary 8.9 up to adding a direct sum of copies of the sheafified free resolution of the residue class field of $S$. In most cases, these summands cannot occur.

Theorem 8.10. Let $\mathcal{L}$ be a monad for a coherent sheaf $\mathcal{F}$ on $\mathbf{P}^{n}$, and let $\mu=$ $\left(m_{0} \leq \cdots \leq m_{n}\right)$. If $\mathcal{L}^{i}=0$ for $|i|>n$ and the terms $\mathcal{L}^{i}=\bigoplus_{j} \mathcal{O}\left(-a_{i j}\right)$ satisfy $m_{i} \leq a_{i j}+i \leq m_{i+n}$ for all $i, j$, then $\mathcal{L}$ is isomorphic to the direct sum of $\mathcal{L}_{\mu}(\mathcal{F})$ and a sum $\mathcal{A}=\bigoplus_{i=1}^{n} \widetilde{\mathbf{L}}\left(\omega_{E}^{r_{i}}\left(-m_{i}\right)[-i]\right)$ of twisted Koszul complexes. Moreover, $r_{i}$ can be nonzero only if $m_{i-1}=m_{i}$. In particular, if the $m_{i}$ are strictly increasing, or if we assume that no direct summand of $\mathcal{L}$ is a monad for $\mathcal{F}$, then $\mathcal{L} \cong \mathcal{L}_{\mu}(\mathcal{F})$.

Proof. Set $L=\Gamma_{*} \mathcal{L}$, and let $\mathcal{K}^{i}$ and $\mathcal{B}^{i}$ be the kernel and the image of the differential $d^{i}: \mathcal{L}^{i} \rightarrow \mathcal{L}^{i+1}$, respectively. We begin by identifying the homology of $L$. Note that $\left(L^{i}\right)_{d}=0$ for $d<m_{i}-i$, so $\left(\mathbf{H}^{i} L\right)_{d}=0$ for $d<m_{i}-i$ too.

Since $\mathcal{L}$ is exact at $\mathcal{L}^{-i}$ for $i>0$, and $\mathcal{L}^{-n-1}=0$, we can use the sequences $0 \rightarrow \mathcal{B}^{-i-1} \rightarrow \mathcal{L}^{-i} \rightarrow \mathcal{B}^{-i} \rightarrow 0$ to show that $\mathbf{H}^{1} \mathcal{B}^{-i}(d)=0$ for $i \geq 2$ and all $d$. Thus $\mathbf{H}^{0} \mathcal{L}^{-j}(d)$ surjects onto $\mathbf{H}^{0} \mathcal{B}^{-j}(d)$ for $j \geq 1$ and all $d$. It follows that $\mathbf{H}^{-i}(L)=0$ for $i>0$, while $\mathbf{H}^{0}(L)$ is the cokernel of $\bigoplus_{d} \mathbf{H}^{0} \mathcal{B}^{-1}(d) \rightarrow \bigoplus_{d} \mathbf{H}^{0} \mathcal{K}^{0}(d)$.

For $0 \leq i<n$ the space $\mathbf{H}^{i+1} \mathcal{B}^{-1}(d)$ injects into $\mathbf{H}^{n} \mathcal{B}^{i-n}(d)$. But $\mathbf{H}^{n} \mathcal{B}^{i-n}$ is the image of $\mathbf{H}^{n} \mathcal{L}^{i-n}$; the hypothesis on the $a_{i j}$ implies that this cohomology vanishes for $d \geq m_{i}-i$. In particular, $\mathbf{H}^{1} \mathcal{B}^{-1}(d)=0$ for $d \geq m_{0}$, and it follows that $\mathbf{H}^{0} L=\bigoplus_{d \geq m_{0}} \mathbf{H}^{0} \mathcal{F}(d)$. 
We next prove that for each $i>0$ there is a short exact sequence

$$
0 \rightarrow k^{r_{i}}\left(m_{i}-i\right) \rightarrow \mathbf{H}^{i}(L) \rightarrow \bigoplus_{d \geq m_{i}-i} \mathbf{H}^{i} \mathcal{F}(d) \rightarrow 0,
$$

where $r_{i}=0$ unless $m_{i-1}=m_{i}$. In fact we shall identify this sequence with the direct sum, over $d \geq m_{i}-i$, of the sequences

$$
\mathbf{H}^{i} \mathcal{B}^{-1}(d) \rightarrow \mathbf{H}^{i} \mathcal{K}^{0}(d) \rightarrow \mathbf{H}^{i} \mathcal{F}(d) \rightarrow \mathbf{H}^{i+1} \mathcal{B}^{-1}(d),
$$

which come from the sequence $0 \rightarrow \mathcal{B}^{-1} \rightarrow \mathcal{K}^{0} \rightarrow \mathcal{F} \rightarrow 0$ expressing the fact that $\mathcal{L}$ is a monad for $\mathcal{F}$.

We have already seen that $\mathbf{H}^{i+1} \mathcal{B}^{-1}(d)=0$ for $d \geq m_{i}-i$. It follows that the right-hand term of $(*)$ is 0 for $d \geq m_{i}-i$, and the left-hand term is 0 unless $m_{i}=m_{i-1}$, in which case it is $k^{r_{i}}$, where $r_{i}=\mathrm{h}^{i} \mathcal{B}^{-1}\left(m_{i-1}-(i-1)\right)$.

From the long exact sequences in cohomology - and, in case $i=n=1$, the hypothesis on the $a_{1, j}$-we see that $\mathbf{H}^{i} L=\bigoplus_{d \geq m_{i}-i} \mathbf{H}^{1} \mathcal{K}^{i-1}(d)$. For all $0<i \leq n$ we have

$$
\bigoplus_{d \geq m_{i}-i} \mathbf{H}^{1} \mathcal{K}^{i-1}(d)=\bigoplus_{d \geq m_{i}-i} \mathbf{H}^{i} \mathcal{K}^{0}(d)
$$

These identifications and vanishing identify the two exact sequences as required.

Set $\mathbf{T}^{\prime}=\min \mathbf{R}(L)$ so that $L=\min \mathbf{L}\left(\mathbf{T}^{\prime}\right)$ by Theorem 8.1. Since $\mathbf{T}^{\prime}$ is a complex of free $E$-modules which coincides with the exact complex $\mathbf{T}(\mathcal{F})$ in large cohomological degrees, we can construct a map of complexes $\mathbf{T}^{\prime} \rightarrow \mathbf{T}(\mathcal{F})$.

By Corollary 3.6 we have lin $\mathbf{T}^{\prime}=\bigoplus_{i} \mathbf{R}\left(\mathbf{H}^{i}(L)\right)$. By the first part of the proof, the terms of lin $\mathbf{T}^{\prime}$ can be nonzero only in the range of (internal and cohomological) degrees where $\operatorname{lin} \mathbf{T}_{\mu}$ is equal to $\mathbf{T}$. Hence $\mathbf{T}^{\prime}$ is mapped to $\mathbf{T}_{\mu}$, and we obtain a morphism of monads from the composition $L=\min \mathbf{L}\left(\mathbf{T}^{\prime}\right) \rightarrow \mathbf{L}\left(\mathbf{T}^{\prime}\right) \rightarrow \mathbf{L}\left(\mathbf{T}_{\mu}\right) \rightarrow$ $L_{\mu}$. The morphism of monads induces an isomorphism in homology $\mathcal{F}=\mathbf{H}^{0}(\mathcal{L}) \rightarrow$ $\mathbf{H}^{0}\left(\mathcal{L}_{\mu}\right)=\mathcal{F}$, because by Theorem 8.1 $\mathbf{T}^{\prime}$ and $\mathbf{T}_{\mu}$ coincide in large cohomological degrees.

The induced map $\mathbf{H}^{i}(L) \rightarrow \mathbf{H}^{i}\left(L_{\mu}\right)$ is the surjection of the first part of the proof. Hence the map $\mathbf{T}^{\prime} \rightarrow \mathbf{T}_{\mu}$ is onto. Its kernel has terms $\omega_{E}^{r_{i}}\left(-m_{i}\right)[-i]$, and degree considerations show that it is a trivial complex. Because these terms occur in degrees where $\mathbf{T}_{\mu}$ coincides with the acyclic complex $\mathbf{T}$, the differential of $\mathbf{T}^{\prime}$ carries the generators of these modules into boundaries of $\mathbf{T}_{\mu}$. Thus, after a change of generators in $\mathbf{T}^{\prime}$, we see that $\mathbf{T}^{\prime}$ is the direct sum of $\mathbf{T}_{\mu}$ and the trivial complex $\bigoplus_{i=1}^{n} \omega_{E}^{r_{i}}\left(-m_{i}\right)[-i]$.

Example 8.11. Beilinson's free monad. Beilinson's free monad $\mathcal{B}$ for $\mathcal{F}$ with terms

$$
\mathcal{B}^{i}=\bigoplus_{p} \mathbf{H}^{i-p}\left(\Omega^{p}(p) \otimes \mathcal{F}\right) \otimes \mathcal{O}(-p)
$$

is the partition monad for $\mu=(0,1, \ldots, n)$. This follows from Theorem 8.10.

Example 8.12. Walter's monads. Let $c$ be an integer, and let $\mathcal{F}$ be a sheaf such that $\sum_{e} \mathbf{H}^{i} \mathcal{F}(e)$ is finitely generated for $i \leq c$. Choose

$$
m_{0}<m_{1}<\cdots<m_{c}<<0<<m_{c+1}<\cdots<m_{n}
$$

such that $\mathbf{H}^{i} \mathcal{F}(m-i)=0$ for $m<m_{i}$ and $i \leq c$, and $\mathbf{H}^{i} \mathcal{F}(m-i)=0$ for $m \geq m_{i}$ and $i>c$. The monad $\mathcal{W}=\mathcal{W}(\mathcal{F}, c)=\mathcal{L}_{\mu}(\mathcal{F})$ does not depend on the precise 
values of the $m_{i}$, and hence has only terms

$$
0 \rightarrow \mathcal{W}^{c-n+1} \rightarrow \cdots \rightarrow \mathcal{W}^{0} \rightarrow \cdots \rightarrow \mathcal{W}^{c} \rightarrow 0
$$

by Proposition 8.9. By Theorem $8.10 \mathcal{W}(\mathcal{F}, c)$ is the unique minimal free monad of $\mathcal{F}$ with nonzero components only from $c-n+1$ up to $c$. Thus $\mathcal{W}(\mathcal{F}, c)$ is the monad constructed by Walter Wal] with cohomology $\mathbf{H}^{i}\left(\Gamma_{*} \mathcal{W}\right)=\sum_{e} \mathbf{H}^{i} \mathcal{F}(e)$ for $i=$ $0, \ldots, c$ and zero otherwise.

Example 8.13. Consider a smooth rational surface $X \subset \mathbf{P}^{4}$ of degree $d=11$ and sectional genus 10. The existence of three families of rational surfaces with these invariants is known; see Schreyer [Sch] or Decker and Schreyer [DS2]. The Tate resolution of the ideal sheaf of these surfaces has shape

$$
\begin{array}{ccccccccc}
* * & 1 & . & . & . & . & . & . & \ldots \\
* * & 39 & 30 & 10 & . & . & . & . & \ldots \\
\ldots & . & . & . & 2 & . & . & . & \ldots \\
\ldots & . & . & . & 1 & 5 & 5 & . & \ldots \\
\ldots & . & . & . & . & . & 5 & 32 & * *
\end{array}
$$

with $h^{0} \mathcal{I}_{X}(6)=32$ as a reference point. We display four monads for $\mathcal{I}_{X}$. The first two monads are monads with only 4 terms:

$$
\begin{aligned}
& \mathcal{W}\left(I_{X}, 2\right): \quad \mathcal{O}(-4)^{10} \rightarrow \mathcal{O}(-3)^{20} \rightarrow \mathcal{O}(-2)^{11} \rightarrow \mathcal{O}(-1)^{2}, \\
& \mathcal{W}\left(I_{X}, 1\right): \quad \mathcal{O}(-6)^{2} \rightarrow \mathcal{O}(-5)^{10} \rightarrow \mathcal{O}(-4)^{10} \rightarrow \mathcal{O}(-2)
\end{aligned}
$$

The first monad is linear because $\mathcal{W}\left(I_{X}, 2\right)=\mathcal{L}\left(\mathbf{T}^{\geq 2}\right)$. The two following monads are somewhat more complicated and hence less convenient:

$$
\begin{aligned}
& \mathcal{L}\left(\mathbf{T}^{\geq 4}\right): \quad \mathcal{O}(-7)^{1} \rightarrow \quad \mathcal{O}(-6)^{7} \rightarrow \quad \mathcal{O}(-5)^{20} \rightarrow \mathcal{O}(-4)^{20} \rightarrow \mathcal{O}(-3)^{5},
\end{aligned}
$$

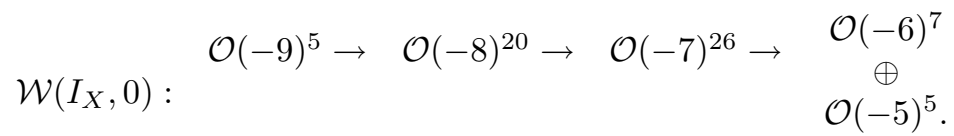

These monads, up to twist, are Beilinson monads for $\mathcal{I}_{X}(m)$ for $m=1,2,3$ and 5 , respectively. The construction of such surfaces in [Sch] was done by a computer search for monads of shape $\mathcal{W}\left(I_{X}, 1\right)$.

Remark 8.14. The degree of smooth rational surfaces in $\mathbf{P}^{4}$ is bounded, according to Ellingsrud and Peskine [EPe. Smooth rational surfaces with sectional genus $\pi>0$ (this excludes the cubic scroll and the projected Veronese surface) have a linear Walter monad. Indeed, by Severi's theorem $\mathbf{H}^{1}\left(I_{X}(1)\right)=0$ and hence $\mathcal{W}\left(I_{X}, 2\right)=\mathcal{L}\left(\mathbf{T}^{\geq 2}\right)$. The numerical type of these monads is

$$
\mathcal{O}(-4)^{\pi} \rightarrow \mathcal{O}(-3)^{2 \pi+s-2} \rightarrow \mathcal{O}(-2)^{\pi+2 s-3} \rightarrow \mathcal{O}(-1)^{s}
$$

with $s=h^{1} \mathcal{O}_{X}(1)=\pi-d+3$.

The conjectured bound is $d \leq 15$. Perhaps even $d \leq 11$ is true. However, at present the best known bound is $d \leq 52$; see Decker and Schreyer [DS2]. 


\section{REFERENCES}

[ABW] K. Akin, D. A. Buchsbaum, and J. Weyman: Schur functors and Schur complexes. Adv. in Math. 44 (1982) 207-278. MR 84c:20021

[AO] V. Ancona and G. Ottaviani: An introduction to the derived categories and the theorem of Beilinson. Atti Accademia Peloritana dei Pericolanti, Classe I de Scienze Fis. Mat. et Nat. LXVII (1989) 99-110. MR 92g:14013

[AAH] A. Aramova, L. A. Avramov, J. Herzog: Resolutions of monomial ideals and cohomology over exterior algebras. Trans. Amer. Math. Soc. 352 (2000) 579-594. MR 2000c:13021

[Bar] W. Barth: Moduli of vector bundles on the projective plane. Invent. Math. 42 (1977) 63-91. MR 57:324

[Bei] A. Beilinson: Coherent sheaves on $\mathbf{P}^{n}$ and problems of linear algebra. Funct. Anal. and its Appl. 12 (1978) 214-216. (Trans. from Funkz. Anal. i. Ego Priloz. 12 (1978) 68-69.) MR 80c: $14010 b$

[BGG] I. N. Bernstein, I. M. Gel'fand and S. I. Gel'fand: Algebraic bundles on $\mathbf{P}^{n}$ and problems of linear algebra. Funct. Anal. and its Appl. 12 (1978) 212-214. (Trans. from Funkz. Anal. i. Ego Priloz 12 (1978) 66-67.) MR 80c:14010a

[BE] D. A. Buchsbaum and D. Eisenbud: Generic free resolutions and a family of generically perfect ideals. Adv. Math. 18 (1975) 245-301. MR 53:391

[Buc1] R.-O. Buchweitz: Appendix to Cohen-Macaulay modules on quadrics, by R.-O. Buchweitz, D. Eisenbud, and J. Herzog. In Singularities, representation of algebras, and vector bundles (Lambrecht, 1985), Springer-Verlag Lecture Notes in Math. 1273 (1987) 96-116. MR 88h:14001

[Buc2] R.-O. Buchweitz: Maximal Cohen-Macaulay modules and Tate-Cohomology over Gorenstein ring Preprint (1985).

[DE] W. Decker and D. Eisenbud: Sheaf algorithms using the exterior algebra, in Computations in Algebraic Geometry with Macaulay2, ed. D. Eisenbud, D. Grayson, M. Stillman, and B. Sturmfels. Springer-Verlag, New York, 2001.

[DS1] W. Decker and F.-O. Schreyer: On the uniqueness of the Horrocks-Mumford bundle. Math. Ann. 273 (1986) 415-443. MR 87d:14009

[DS2] W. Decker and F.-O. Schreyer: Non-general type surfaces in $\mathbf{P}^{4}$ : Some remarks on bounds and constructions. J. Symbolic Comp. 29 (2000), 545-582. MR 2002a:14064

[Dec] W. Decker: Stable rank 2 bundles with Chern-classes $c_{1}=-1, c_{2}=4$. Math. Ann. 275 (1986) 481-500. MR 87j:14017

[Eis] D. Eisenbud: Commutative Algebra with a View Toward Algebraic Geometry. SpringerVerlag, New York, 1995. MR 97a:13001

[EG] D. Eisenbud and S. Goto: Linear free resolutions and minimal multiplicity. J. Algebra 88 (1984) 89-133. MR 85f:13023

[EPo] D. Eisenbud and S. Popescu: Gale Duality and Free Resolutions of Ideals of Points. Invent. Math. 136 (1999) 419-449. MR 2000i:13014

[EPSW] D. Eisenbud, S. Popescu, F.-O. Schreyer and C. Walter: Exterior algebra methods for the Minimal Resolution Conjecture, Duke Math. J. 112 (2002), 379-395.

[EPY] D. Eisenbud, S. Popescu, and S. Yuzvinsky: Hyperplane arrangements and resolutions of monomial ideals over an exterior algebra. Trans. Amer. Math. Soc., this issue.

[ES1] D. Eisenbud and F.-O. Schreyer: Sheaf cohomology and free resolutions over the exterior algebras, http://arXiv.org/abs/math.AG/0005055 Preprint (2000).

[ES2] D. Eisenbud, F.-O. Schreyer, and Jerzy Weyman: Resultants and Chow forms via exterior syzygies. J. Amer. Math. Soc. 16 (2003) 537-579.

[EPe] G. Ellingsrud and C. Peskine: Sur le surfaces lisse de $\mathbf{P}^{4}$. Invent. Math. 95 (1989) 1-12. MR 89j:14023

[EW] D. Eisenbud and J. Weyman: Fitting's Lemma for $\mathbb{Z} / 2$-graded modules. Trans. Amer. Math. Soc., this issue.

[Flo1] G. Fløystad: Koszul duality and equivalences of categories. http://arXiv.org/ abs/math.RA/0012264 Preprint (2000a).

[Flo2] G. Fløystad: Describing coherent sheaves on projective spaces via Koszul duality. http://arXiv.org/abs/math.RA/0012263 Preprint (2000b).

[Flo3] G. Fløystad: Monads on projective spaces. Communications in Algebra. 28 (2000c) 55035516. MR 2002e:14070 
[Gel] S. I. Gel'fand: Sheaves on $\mathbf{P}^{n}$ and problems in linear algebra. Appendix to the Russian edition of [OSS], Mir, Moscow, 1984. MR 86i:14005

[GM] S. I. Gel'fand and Yu. I. Manin: Methods of Homological Algebra. Springer-Verlag, New York, 1996. MR 97j:18001

[GS] D. Grayson and M. Stillman: Macaulay2. http://www.math.uiuc.edu/Macaulay2/.

[Gre] M. Green: The Eisenbud-Koh-Stillman Conjecture on Linear Syzygies. Invent. Math. 136 (1999) 411-418. MR 2000j:13024

[GD] A. Grothendieck and J. Dieudonné: Éléments de la Géometrie Algébrique IV: Étude locale des schémas et de morphismes de schémas (deuxième partie). Inst. Hautes Etudes Sci. Publ. Math. 24 (1965). MR 33:7330

[Hap] D. Happel: Triangulated Categories in the Representation Theory of Finite-Dimensional Algebras. London Math. Soc. Lecture Notes Ser. 119, 1988. MR 89e:16035

[Har] R. Hartshorne: Algebraic geometry, Springer Verlag, 1977. MR 57:3116

[HR] J. Herzog and T. Römer: Resolutions of modules over the exterior algebra, working notes, 1999.

[Hor] G. Horrocks: Vector bundles on the punctured spectrum of a local ring, Proc. London Math. Soc. (3) 14 (1964), 714-718. MR 30:121

[HM] G. Horrocks and D. Mumford: A rank 2 vector bundle on $\mathbf{P}^{4}$ with 15,000 symmetries. Topology 12 (1973) 63-81. MR 52:3164

[Kap1] M. M. Kapranov: On the derived categories of coherent sheaves on some homogeneous spaces. Invent. Math. 92 (1988) 479-508. MR 89g:18018

[Kap2] M. M. Kapranov: On the derived category and $K$-functor of coherent sheaves on intersections of quadrics. Izv. Akad. Nauk SSSR Ser. Mat. 52 (1988) 186-199; English transl., Math. USSR Izv. 32 (1989), 191-204. MR 89f:14014

[MP] M. Martin-Deschamps and D. Perrin: Sur la classification des courbes gauches. Astérisque 184-185 (1990). MR 91h:14039

[OSS] C. Okonek, M. Schneider, and H. Spindler: Vector Bundles on Complex Projective Spaces. Birkhäuser, Boston 1980. MR 81b:14001

[Orl] D. O. Orlov: Projective bundles, monoidal transformations, and derived categories of coherent sheaves. Izv. Ross. Akad. Nauk Ser. Mat. 56 (1992) 852-862; translation in Russian Acad. Sci. Izv. Math. 41 (1993) 133-141. MR 94e:14024

[Pri] S. B. Priddy: Koszul resolutions. Trans. Amer. Math. Soc. 152 (1970) 39-60. MR 42:346

[Rao] P. Rao: Liaison equivalence classes, Math. Ann. 258 (1981) 169-173. MR 83j:14045

[Sch] F.-O. Schreyer: Small fields in constructive algebraic geometry. In S. Maruyama ed.: Moduli of Vector Bundles, Sanda 1994. New York, Dekker 1996, 221-228. MR 97h:14050

[Swa] R. G. Swan: K-theory of quadric hypersurfaces. Ann. of Math. 122 (1985) 113-153. MR 87g:14006

[Wal] C. Walter: Algebraic cohomology methods for the normal bundle of algebraic space curves. Preprint (1990).

Department of Mathematics, University of California Berkeley, Berkeley, CaliforNIA 94720

E-mail address: eisenbud@math.berkeley.edu

Mathematisk Institutt, Johs. Brunsgt. 12, N-5008 Bergen, Norway

E-mail address: gunnar@mi.uib.no

FB Mathematik, Universität Bayreuth D-95440 Bayreuth, Germany

E-mail address: schreyer@btm8x5.mat.uni-bayreuth.de 